\title{
Regression-based complexity reduction of the nested Monte Carlo methods*
}

\author{
Denis Belomestny ${ }^{\dagger} \quad$ Stefan Häfner ${ }^{\ddagger} \quad$ Mikhail Urusov ${ }^{\S}$
}

\begin{abstract}
In this paper we propose a novel dual regression-based approach for pricing American options. This approach reduces the complexity of the nested Monte Carlo method and has especially simple form for time discretized diffusion processes. We analyse the complexity of the proposed approach both in the case of fixed and increasing number of exercise dates. The method is illustrated by several numerical examples.

Keywords: Bermudan options, Monte Carlo methods, nested simulations, control variates, regression methods
\end{abstract}

AMS subject classifications: $65 \mathrm{C} 05,60 \mathrm{H} 35,62 \mathrm{P} 05$

\section{Introduction}

In contrast to European options, which may be exercised only at a fixed date, an American option grants its holder the right to select the time at which to exercise the option. A general class of American option pricing problems can be formulated through an $\mathbb{R}^{d}$-valued $\left(\mathcal{F}_{t}\right)$-Markov process $\left(X_{t}\right)_{t \in[0, T]}$ with a deterministic starting point $X_{0}=x_{0} \in \mathbb{R}^{d}$ defined on a filtered probability space $\left(\Omega, \mathcal{F},\left(\mathcal{F}_{t}\right)_{0 \leq t \leq T}, \mathrm{P}\right)$. Let us recall that each $\mathcal{F}_{t}$ is a $\sigma$-algebra of subsets of $\Omega$, and $\mathcal{F}_{s} \subseteq \mathcal{F}_{t} \subseteq \mathcal{F}$ for $s \leq t$. We first consider options admitting a finite set of exercise opportunities $0=t_{0}<t_{1}<t_{2}<\ldots<t_{J}=T$, called Bermudan options, with corresponding Markov chain

$$
X_{j}:=X_{t_{j}}, \quad j=0, \ldots, J .
$$

This option pays $g_{j}\left(X_{j}\right)$, if exercised at time $t_{j}, j=0, \ldots, J$, for some known Borel-measurable functions $g_{0}, \ldots, g_{J}$ mapping $\mathbb{R}^{d}$ into $[0, \infty)$. Below we assume that $g_{j}\left(X_{j}\right) \in L^{2}$ for all $j$. Let $\mathcal{T}_{j}$ denote the set of stopping times taking values in $\{j, j+1, \ldots, J\}$. As a standard result in the theory of contingent claims, the equilibrium price $v_{j}^{*}(x)$ of the Bermudan option at time $t_{j}$ in state $x$, given that the option was not exercised prior to $t_{j}$, is its value under the optimal exercise policy

$$
v_{j}^{*}(x)=\sup _{\tau \in \mathcal{T}_{j}} \mathrm{E}\left[g_{\tau}\left(X_{\tau}\right) \mid X_{j}=x\right], \quad x \in \mathbb{R}^{d} .
$$

*Stefan Häfner thanks the Faculty of Mathematics of the University of Duisburg-Essen, where this work was carried out.

${ }^{\dagger}$ University of Duisburg-Essen, Essen, Germany; E-mail address: denis.belomestny@uni-due.de

$\ddagger$ PricewaterhouseCoopers GmbH, Düsseldorf, Germany; E-mail address: stefan.haefner@pwc.com

$\S$ University of Duisburg-Essen, Essen, Germany; E-mail address: mikhail.urusov@uni-due.de 
Clearly, any given stopping rules $\tau_{j} \in \mathcal{T}_{j}$ are generally suboptimal and give us lower bounds

$$
v_{j}(x):=\mathrm{E}\left[g_{\tau_{j}}\left(X_{\tau_{j}}\right) \mid X_{j}=x\right] \leq v_{j}^{*}(x), \quad j=0, \ldots, J,
$$

for the option price.

By now, there are well established algorithms that produce tight lower bounds for prices of Bermudan options. For instance, the computationally efficient Longstaff and Schwartz [14] algorithm based on nonparametric regression is widely used. It is crucial especially in highdimensional problems, where explicit formulas for option prices are typically unavailable even in the simplest Black-Scholes framework. Moreover, in addition to good lower bounds it is important to have tight upper bounds for the prices because only in the case when we have both bounds, we have reliable confidence intervals for the unknown true price as well as we know the magnitude of the error in our approximations. Even when an estimate of the variance of a lower bound for the option price is available, we usually cannot construct a reliable confidence interval for the unknown true option price because we typically do not know the magnitude of the bias. An upper bound for the true price could be generated from any given exercise policy using the following dual approach, which was proposed in Rogers [16] and Haugh and Kogan [12]. For any $0 \leq i \leq J$ and any supermartingale $\left(Y_{j}\right)_{i \leq j \leq J}$ with $Y_{i}=0$, it holds

$$
\begin{aligned}
v_{i}^{*}\left(X_{i}\right)=\sup _{\tau \in \mathcal{T}_{i}} \mathrm{E}\left[g_{\tau}\left(X_{\tau}\right) \mid \mathcal{F}_{i}\right] & \leq \sup _{\tau \in \mathcal{T}_{i}} \mathrm{E}\left[g_{\tau}\left(X_{\tau}\right)-Y_{\tau} \mid \mathcal{F}_{i}\right] \\
& \leq \mathrm{E}\left[\max _{i \leq j \leq J}\left(g_{j}\left(X_{j}\right)-Y_{j}\right) \mid \mathcal{F}_{i}\right]
\end{aligned}
$$

(we now use the shorthand $\mathcal{F}_{j}:=\mathcal{F}_{t_{j}}$ ). Therefore the right-hand side of (1.1) provides an upper bound for $v_{i}^{*}\left(X_{i}\right)$. It can be derived that both inequalities in (1.1) are equalities for the martingale part of the Doob-Meyer decomposition of the price process $\left(v_{j}^{*}\left(X_{j}\right)\right)_{i \leq j \leq J}$

$$
Y_{i}^{*}=0, \quad Y_{j}^{*}=\sum_{l=i+1}^{j}\left(v_{l}^{*}\left(X_{l}\right)-\mathrm{E}\left[v_{l}^{*}\left(X_{l}\right) \mid \mathcal{F}_{l-1}\right]\right), \quad j=i+1, \ldots, J .
$$

In fact, $Y^{*}$ satisfies the following even stronger almost sure identity

$$
v_{i}^{*}\left(X_{i}\right)=\max _{i \leq j \leq J}\left(g_{j}\left(X_{j}\right)-Y_{j}^{*}\right), \quad \text { a.s. }
$$

(see [17]). The duality representation provides a simple way to estimate the Snell envelope from above, using approximations $\left(v_{i}\left(X_{i}\right)\right)$ for the value functions $\left(v_{i}^{*}\left(X_{i}\right)\right)$. Let $Y$ be a martingale defined via

$$
Y_{0}=0, \quad Y_{j}=\sum_{l=1}^{j}\left(v_{l}\left(X_{l}\right)-\mathrm{E}\left[v_{l}\left(X_{l}\right) \mid \mathcal{F}_{l-1}\right]\right), \quad j=1, \ldots, J .
$$

Then, for $i=0$, we get that

$$
V_{0}:=v_{0}\left(x_{0}\right)=\mathrm{E}\left[\max _{0 \leq j \leq J}\left(g_{j}\left(X_{j}\right)-Y_{j}\right)\right]
$$

is an upper bound for $V_{0}^{*}:=v_{0}^{*}\left(x_{0}\right)$.

Another approach to construct upper bounds is based on the so-called discrete time early exercise premium representation

$$
v_{0}^{*}\left(x_{0}\right)=\mathrm{E}\left[g_{J}\left(X_{J}\right)+\sum_{l=1}^{J}\left(g_{l-1}\left(X_{l-1}\right)-\mathrm{E}\left[v_{l}^{*}\left(X_{l}\right) \mid \mathcal{F}_{l-1}\right]\right)^{+}\right],
$$


which was first established in [5]. Then, for any lower approximation $v_{l}\left(X_{l}\right)$ with $v_{l}\left(X_{l}\right) \leq$ $v_{l}^{*}\left(X_{l}\right), l=1, \ldots, J$, a.s., we get an upper bound

$$
U_{0}=\mathrm{E}\left[g_{J}\left(X_{J}\right)+\sum_{l=1}^{J}\left(g_{l-1}\left(X_{l-1}\right)-\mathrm{E}\left[v_{l}\left(X_{l}\right) \mid \mathcal{F}_{l-1}\right]\right)^{+}\right],
$$

i.e. $U_{0} \geq V_{0}^{*}$. There are examples, where the upper bound $U_{0}$ is more accurate than the dual upper bound $V_{0}$, and there are opposite examples (see Section 2.4 in [4]).

In this paper, we suggest a novel nonparametric regression algorithm to construct computationally efficient approximations for the conditional expectations involved in (1.3) and (1.5). Nonparametric regression algorithms like that of Longstaff and Schwartz have become among the most successful and widely used methods for approximating the values of American-style (Bermudan) options, in particular for high-dimensional problems. Due to their popularity, the analysis of the convergence properties of these types of Monte Carlo algorithms is a problem of fundamental importance in applied probability and mathematical finance, see e.g. Clément, Lamberton and Protter [8], Zanger [20] and references therein. Here we rigorously analyse the convergence properties of the proposed regression algorithm and derive its complexity. To this end, we first establish in Section 2 a new $L^{2}$ error bound for the nested simulations approach based either on (1.4) or on (1.5), which turns out to be instrumental both for understanding how to improve the standard nested estimators and for the error analysis of the proposed algorithm (the latter is constructed and studied in detail in later sections). The performance of our algorithm is illustrated by the example of max-call Bermudan options.

\section{Nested simulations approach}

The nested simulations approach for computing $V_{0}$ of $(1.4)$ relies on the approximation of the conditional expectations in (1.3) via (nested) Monte Carlo. This approach was first proposed in Andersen and Broadie [1] for the computation of the dual upper bound (1.4). Let us describe this method in more detail. Fix some natural numbers $N_{d}$ and $N$. The dual nested simulations approach consists in using the estimate

$$
V_{N, N_{d}}=\frac{1}{N} \sum_{n=1}^{N}\left[\max _{0 \leq j \leq J}\left(g_{j}\left(X_{j}^{(n)}\right)-Y_{j, n, N_{d}}\right)\right],
$$

where

$$
Y_{j, n, N_{d}}=\sum_{l=1}^{j}\left(v_{l}\left(X_{l}^{(n)}\right)-\frac{1}{N_{d}} \sum_{n_{d}=1}^{N_{d}} v_{l}\left(X_{l}^{\left(n_{d}, n\right)}\right)\right), \quad j=0, \ldots, J,
$$

$\left(\sum_{1}^{0}:=0\right),\left(X_{l}^{(1)}, \ldots, X_{l}^{(N)}\right)$ is a sample from the distribution of $X_{l}$, and, for any fixed $n$, the sample $X_{l}^{(1, n)}, \ldots, X_{l}^{\left(N_{d}, n\right)}$ is drawn from the conditional distribution of $X_{l}$ given $X_{l-1}=X_{l-1}^{(n)}$. As an estimate for $V_{0}$ the random variable $V_{N, N_{d}}$ is biased high (see (2.1) below). The next theorem presents a bound for its mean squared error (MSE).

Theorem 2.1. We have for the estimator $V_{N, N_{d}}$

$$
\mathrm{E} V_{N, N_{d}} \geq V_{0}
$$


i.e. it is an upper bound for $V_{0}$ and hence for $V_{0}^{*}$. Moreover, it holds

$$
\begin{aligned}
& \mathrm{E}\left[\left(V_{N, N_{d}}-V_{0}\right)^{2}\right] \\
& \leq \frac{4}{N_{d}}\left(1+\frac{1}{N}\right) \sum_{l=1}^{J} \mathrm{E}\left[\operatorname{Var}\left[v_{l}\left(X_{l}\right) \mid X_{l-1}\right]\right]+\frac{4}{N} \sum_{l=1}^{J} \mathrm{E}\left[\operatorname{Var}\left[v_{l}^{*}\left(X_{l}\right)-v_{l}\left(X_{l}\right) \mid X_{l-1}\right]\right] \\
& \leq \frac{4}{N_{d}}\left(1+\frac{1}{N}\right) \sum_{l=1}^{J} \mathrm{E}\left[\operatorname{Var}\left[v_{l}\left(X_{l}\right) \mid X_{l-1}\right]\right]+\frac{4}{N} \sum_{l=1}^{J} \mathrm{E}\left[\left(v_{l}^{*}\left(X_{l}\right)-v_{l}\left(X_{l}\right)\right)^{2}\right] .
\end{aligned}
$$

Statement (2.1) is known (see Remark 3.2 in [6]) and presented here only to make the exposition self-contained. On the other hand, bound (2.2) for the MSE of $V_{N, N_{d}}$ is new. Several related quantities were extensively studied in Chen and Glasserman [7] and Belomestny et al [6], but neither of these papers contains such a bound.

Inequality 2.2) is surprising because none of $V_{N, N_{d}}$ or $V_{0}$ involves the real price $\left(v_{l}^{*}\left(X_{l}\right)\right)$. Conceptually, the real price comes into play as it is inherent in the optimal stopping problem for $\left(g_{j}\left(X_{j}\right)\right)$ (notice that $\left(g_{j}\left(X_{j}\right)\right)$ constitutes our problem data). On the technical side, the real price appears in the proof due to the almost sure property (1.2). More precisely, the random variable $\max _{0 \leq j \leq J}\left(g_{j}\left(X_{j}\right)-Y_{j}^{*}\right)$, being degenerate, can be introduced into 7.3 without changing the variance term in $(7.3)$.

Remark 2.2. (i) Bound (2.2) is very informative, as it not only gives an error estimate for $V_{N, N_{d}}$, but also shows ways to improve the quality of $V_{N, N_{d}}$. While the second term in the r.h.s. of (2.2) can be reduced by making the bound $v_{l}$ closer to $v_{l}^{*}$, the first one can be made smaller by reducing the magnitude of the conditional variances $\operatorname{Var}\left[v_{l}\left(X_{l}\right) \mid X_{l-1}\right]$.

(ii) In addition to the composite bound (2.2) for the MSE of $V_{N, N_{d}}$ it is instructive to see what in this bound accounts for the squared bias and what for the variance. We will see in the proof of Theorem 2.1 that

$$
\left(\mathrm{E} V_{N, N_{d}}-V_{0}\right)^{2} \leq \frac{4}{N_{d}} \sum_{l=1}^{J} \mathrm{E}\left[\operatorname{Var}\left[v_{l}\left(X_{l}\right) \mid X_{l-1}\right]\right]
$$

and

$$
\operatorname{Var}\left[V_{N, N_{d}}\right] \leq \frac{1}{N} \frac{4}{N_{d}} \sum_{l=1}^{J} \mathrm{E}\left[\operatorname{Var}\left[v_{l}\left(X_{l}\right) \mid X_{l-1}\right]\right]+\frac{4}{N} \sum_{l=1}^{J} \mathrm{E}\left[\operatorname{Var}\left[v_{l}^{*}\left(X_{l}\right)-v_{l}\left(X_{l}\right) \mid X_{l-1}\right]\right]
$$

Roughly speaking, this means that $N$ (resp. $N_{d}$ ) accounts for the variance (resp. the bias) of the estimator $V_{N, N_{d}}$. That is, we need to increase $N$ (resp. $N_{d}$ ) in order to reduce the variance (resp. the bias). On top of that we can observe a more delicate effect that increasing $N_{d}$ alone (i.e. with $N$ being fixed) also reduces a (small) part of the variance of $V_{N, N_{d}}$.

Bound (2.2) for the MSE of $V_{N, N_{d}}$ also enables us to analyse the complexity of the dual nested simulations approach. Since the cost of computing $V_{N, N_{d}}$ is of order $N N_{d}$ (recall that $J$ is fixed for now), the overall complexity of the estimate $V_{N, N_{d}}$, i.e. the minimal cost needed to achieve $\mathrm{E}\left[\left(V_{N, N_{d}}-V_{0}\right)^{2}\right] \leq \varepsilon^{2}$, is of order $\varepsilon^{-4}$. In the next two sections we will develop a regression-based approach, which will result in a significant reduction of the complexity (see Remark 4.2.

Let us mention two relevant modifications of the nested dual algorithm proposed in the literature. Firstly, in Belomestny et al [2] an algorithm not involving sub-simulation was suggested, 
where an approximation for the Doob martingale was constructed using the martingale representation theorem and some approximation of the true price process. However, that method requires an additional discretization of stochastic integrals and suffers from some instability for small discretization steps. Secondly, a multilevel-type algorithm was developed in Belomestny et al [6], which has a similar performance, in terms of complexity, as the algorithm presented in the next sections, but works under very different conditions (e.g. the algorithm in [6] does not take advantage of the smoothness properties of the involved conditional expectations).

In a similar way, a nested simulations estimator $U_{N, N_{d}}$ for $U_{0}$ of 1.5 can be constructed as follows

$$
U_{N, N_{d}}=\frac{1}{N} \sum_{n=1}^{N}\left[g_{J}\left(X_{J}^{(n)}\right)+\sum_{j=1}^{J}\left(g_{j-1}\left(X_{j-1}^{(n)}\right)-Z_{j, n, N_{d}}\right)^{+}\right],
$$

where

$$
Z_{j, n, N_{d}}=\frac{1}{N_{d}} \sum_{n_{d}=1}^{N_{d}} v_{j}\left(X_{j}^{\left(n_{d}, n\right)}\right), \quad j=1, \ldots, J,
$$

where the sample $X_{j}^{(1, n)}, \ldots, X_{j}^{\left(N_{d}, n\right)}$ is drawn from the conditional distribution of $X_{j}$ given $X_{j-1}=X_{j-1}^{(n)}$. In the next proposition, we present a new bound for the MSE of $U_{N, N_{d}}$.

Proposition 2.3. We have for the estimator $U_{N, N_{d}}$

$$
\mathrm{E} U_{N, N_{d}} \geq U_{0}
$$

i.e. it is an upper bound for $U_{0}$ and hence for $V_{0}^{*}$. Moreover, it holds

$$
\begin{aligned}
& \mathrm{E}\left[\left(U_{N, N_{d}}-U_{0}\right)^{2}\right] \\
& \leq \frac{J}{N_{d}} \sum_{j=1}^{J} \mathrm{E}\left[\operatorname{Var}\left[v_{j}\left(X_{j}\right) \mid X_{j-1}\right]\right]+\frac{1}{N} \operatorname{Var}\left[g_{J}\left(X_{J}^{(1)}\right)+\sum_{j=1}^{J}\left(g_{j-1}\left(X_{j-1}^{(1)}\right)-Z_{j, 1, N_{d}}\right)^{+}\right] .
\end{aligned}
$$

Again, bound (2.6) shows a way of improving the quality of the estimator $U_{N, N_{d}}$ by variance reduction technique: the first term on the right-hand side can be made smaller by reducing the magnitude of the conditional variances $\operatorname{Var}\left[v_{l}\left(X_{l}\right) \mid X_{l-1}\right]$. Recall that this also improves the quality of the dual nested estimator $V_{N, N_{d}}$. The second summand on the right-hand side of (2.6) is of order $J^{2} / N$ whenever all functions $g_{j}, j=0, \ldots, J$, are uniformly bounded, but this estimate $J^{2} / N$ is usually somewhat rough. In specific situations the generic bound (2.6) should be complemented with specific bounds for the second term on the right-hand side of (2.6).

\section{Variance reduction via regression}

Usually the process $\left(X_{t}\right)_{t \in[0, T]}$ cannot be simulated exactly, and one has to use some approximation of it. Suppose that, for some $\Delta>0$, the time approximations $X_{\Delta, l \Delta}, l=0, \ldots, L$, with $L=\lfloor T / \Delta\rfloor \geq J$ satisfy the following recurrence relations

$$
X_{\Delta, l \Delta}=\Phi_{l}\left(X_{\Delta,(l-1) \Delta}, \xi_{l}\right), \quad l=1, \ldots, L, \quad X_{\Delta, 0}=x_{0},
$$

for some i.i.d. random vectors $\xi_{l} \in \mathbb{R}^{m}$ with distribution $\mu$ and some Borel-measurable functions $\Phi_{l}: \mathbb{R}^{d+m} \rightarrow \mathbb{R}^{d}$. By $\left(\mathcal{G}_{l}\right)_{l \in\{0, \ldots, L\}}$ we denote the filtration with $\mathcal{G}_{0}=$ triv generated by $\left(\xi_{l}\right)_{l=1, \ldots, L}$. It follows from (3.1) that $\left(X_{\Delta, l \Delta}\right)_{l \in\{0, \ldots, L\}}$ is a $\left(\mathcal{G}_{l}\right)$-Markov process. Let $\left(\phi_{k}\right)_{k \in \mathbb{Z}_{+}}$be a complete orthonormal system in $L^{2}\left(\mathbb{R}^{m}, \mu\right)$ with $\phi_{0} \equiv 1$. In particular,

$$
\mathrm{E}\left[\phi_{i}(\xi) \phi_{j}(\xi)\right]=\delta_{i j}, \quad i, j \in \mathbb{Z}_{+} .
$$


Notice that this implies that the random variables $\phi_{k}(\xi), k \geq 1$, are centered.

The following result can be viewed as a discrete-time analogue of the Clark-Ocone formula or as a conditioned version of Theorem 2.1 in [3].

Theorem 3.1. Consider some $j<p$ in $\{0,1, \ldots, L\}$. It holds for any Borel-measurable function $f$ with $\mathrm{E}\left[\left|f\left(X_{\Delta, p \Delta}\right)\right|^{2}\right]<\infty$

$$
f\left(X_{\Delta, p \Delta}\right)=\mathrm{E}\left[f\left(X_{\Delta, p \Delta}\right) \mid X_{\Delta, j \Delta}\right]+\sum_{k \geq 1} \sum_{l=j+1}^{p} a_{p, l, k}\left(X_{\Delta,(l-1) \Delta}\right) \phi_{k}\left(\xi_{l}\right),
$$

where the series in the r.h.s. converges in $L^{2}$ sense. The coefficients in (3.2) can be computed via

$$
a_{p, l, k}(x)=\mathrm{E}\left[f\left(X_{\Delta, p \Delta}\right) \phi_{k}\left(\xi_{l}\right) \mid X_{\Delta,(l-1) \Delta}=x\right]
$$

for $l \in\{j+1, \ldots, p\}$ and $k \in \mathbb{N}$.

For fixed $j<p$ in $\{0,1, \ldots, L\}$, define

$$
M_{j, p}=\sum_{k \geq 1} \sum_{l=j+1}^{p} a_{p, l, k}\left(X_{\Delta,(l-1) \Delta}\right) \phi_{k}\left(\xi_{l}\right)
$$

and notice that $\mathrm{E}\left[M_{j, p} \mid \mathcal{G}_{j}\right]=0$ a.s. and, in particular, $\mathrm{E}\left[M_{j, p} \mid X_{\Delta, j \Delta}\right]=0$ a.s. Theorem 3.1 implies that

$$
\operatorname{Var}\left[f\left(X_{\Delta, p \Delta}\right)-M_{j, p} \mid X_{\Delta, j \Delta}\right]=0 \quad \text { a.s. }
$$

hence $M_{j, p}$ is a perfect control variate for estimating $\mathrm{E}\left[f\left(X_{\Delta, p \Delta}\right) \mid X_{\Delta, j \Delta}\right]$. In order to use the control variate $M_{j, p}$, we need to compute the coefficients $a_{p, l, k}$. This can be done by using regression in the following way: first we generate $N_{r}$ discretized paths $X_{\Delta, 1 \Delta}^{(n)}, \ldots, X_{\Delta, L \Delta}^{(n)}, n=$ $N+1, \ldots, N+N_{r}$, of the process $X$ (so-called "training paths") and then solve the least squares optimization problems

$$
\hat{a}_{p, l, k}=\underset{\psi \in \operatorname{span}\left(\psi_{1}, \ldots, \psi_{Q}\right)}{\arg \min } \sum_{n=N+1}^{N+N_{r}}\left|f\left(X_{\Delta, p \Delta}^{(n)}\right) \phi_{k}\left(\xi_{l}^{(n)}\right)-\psi\left(X_{\Delta,(l-1) \Delta}^{(n)}\right)\right|^{2},
$$

for $l=j+1, \ldots, p$, where $\psi_{1}, \ldots, \psi_{Q}$ is a set of basis functions on $\mathbb{R}^{d}$. Furthermore, we truncate the summation in (3.3) to get an implementable version of the control variate $M_{j, p}$

$$
\hat{M}_{j, p, K}=\sum_{k=1}^{K} \sum_{l=j+1}^{p} \hat{a}_{p, l, k}\left(X_{\Delta,(l-1) \Delta}\right) \phi_{k}\left(\xi_{l}\right) .
$$

To make clear how to understand (3.4), we remark that the random vectors $\xi_{l}, l=1, \ldots, L$, in (3.4) are independent of the $N_{r}$ training paths $\left(X_{\Delta, l \Delta}^{(n)}\right)$ used to obtain the regression-based estimates $\hat{a}_{p, l, k}$, while the ("testing") path $\left(X_{\Delta, l \Delta}\right)$ in the argument of $\hat{a}_{p, l, k}$ in (3.4) is constructed via those random vectors $\xi_{l}$ according to (3.1) (and hence is independent of the training paths).

Let us note that $\mathrm{E}\left[\hat{M}_{j, p, K} \mid X_{\Delta, j \Delta}\right]=0$ due to the martingale transform structure in (3.4) (recall that $\mathrm{E} \phi_{k}\left(\xi_{l}\right)=0$ for $k \geq 1$ ), i.e. $\hat{M}_{j, p, K}$ is indeed a valid control variate in that it does not introduce any bias. The properties of such a control variate are summarised in the following theorem. 
Theorem 3.2. Consider some $j<p$ in $\{0,1, \ldots, L\}$. Suppose that the function $f$ is uniformly bounded by a constant $F$. By $\tilde{a}_{p, l, k}$ we denote the truncated at the level $F$ estimat 1

$$
\tilde{a}_{p, l, k}(x)=T_{F} \hat{a}_{p, l, k}(x)= \begin{cases}\hat{a}_{p, l, k}(x) & \text { if }\left|\hat{a}_{p, l, k}(x)\right| \leq F, \\ F \operatorname{sgn} \hat{a}_{p, l, k}(x) & \text { otherwise, }\end{cases}
$$

and by $\tilde{M}_{j, p, K}$ the control variate defined like in (3.4) but with $\hat{a}_{p, l, k}$ replaced by $\tilde{a}_{p, l, k}$. Furthermore, assume that, for some $\beta \geq 0$ and $B_{\beta}>0$,

$$
\sum_{k=1}^{\infty} k^{\beta} \sum_{l=j+1}^{p} \mathrm{E}\left[a_{p, l, k}^{2}\left(X_{\Delta,(l-1) \Delta}\right)\right] \leq B_{\beta}
$$

and the set of basis functions $\psi_{1}, \ldots, \psi_{Q}$ is chosen in such a way that, for all $k \in \mathbb{N}$,

$$
\sum_{l=j+1}^{p} \inf _{\psi \in \operatorname{span}\left(\psi_{1}, \ldots, \psi_{Q}\right)} \mathrm{E}\left[\left|a_{p, l, k}\left(X_{\Delta,(l-1) \Delta}\right)-\psi\left(X_{\Delta,(l-1) \Delta}\right)\right|^{2}\right] \leq D_{\kappa} Q^{-\kappa},
$$

for some constants $\kappa \geq 0$ and $D_{\kappa}>0$. Then

$$
\begin{aligned}
\mathrm{E}\left[\operatorname{Var}\left[f\left(X_{\Delta, p \Delta}\right)-\tilde{M}_{j, p, K} \mid X_{\Delta, j \Delta}\right]\right] \leq & \tilde{c} F^{2}(p-j) K \frac{Q\left(\log \left(N_{r}\right)+1\right)}{N_{r}} \\
& +8 D_{\kappa} K Q^{-\kappa}+B_{\beta} K^{-\beta}
\end{aligned}
$$

with some universal constant $\tilde{c}$.

Remark 3.3. Theorem 3.2 simplifies in the case when the random vectors $\xi_{l}$ are discrete with finite number of atoms (e.g. think about a weak approximation of an SDE via scheme (3.1) with discrete random vectors $\xi_{l}$; see Part VI in [13] or Chapter 2 in [15]). In this case, the space $L^{2}\left(\mathbb{R}^{m}, \mu\right)$ is finite-dimensional, and hence the basis $\left(\phi_{k}\right)_{k \in\left\{0,1, \ldots, K_{\max }\right\}}$ is finite consisting of say $K_{\max }+1$ elements (recall that $\phi_{0} \equiv 1$ ). Then we can drop assumption (3.6), while the conclusion (3.8) can be replaced with

$$
\mathrm{E}\left[\operatorname{Var}\left[f\left(X_{\Delta, p \Delta}\right)-\tilde{M}_{j, p, K_{\max }} \mid X_{\Delta, j \Delta}\right]\right] \leq \tilde{c} F^{2}(p-j) K_{\max } \frac{Q\left(\log \left(N_{r}\right)+1\right)}{N_{r}}+8 K_{\max } D_{\kappa} Q^{-\kappa}
$$

\section{Dual upper bounds with reduced complexity}

Next we apply the results of the previous section to the nested simulations of dual upper bounds. For the sake of clarity assume that the exercise times coincide with the discretization time grid for some $\Delta>0$, i.e. $L=J$. Instead of $V_{0}$, which is constructed in (1.4) via the exact process, we are now going to estimate its analogue $V_{\Delta, 0}$ constructed via the discretized process

$$
V_{\Delta, 0}=\mathrm{E}\left[\max _{0 \leq j \leq J}\left(g_{j}\left(X_{\Delta, j \Delta}\right)-Y_{\Delta, j \Delta}\right)\right]
$$

with $Y_{\Delta, j \Delta}=\sum_{l=1}^{j}\left(v_{l}\left(X_{\Delta, l \Delta}\right)-\mathrm{E}\left[v_{l}\left(X_{\Delta, l \Delta}\right) \mid X_{\Delta,(l-1) \Delta}\right]\right)$. For any $j=1, \ldots, J$, we need to compute the conditional expectations $\mathrm{E}\left[v_{j}\left(X_{\Delta, j \Delta}\right) \mid X_{\Delta,(j-1) \Delta}\right]$. By Theorem 3.1, we have the following representation

$$
v_{j}\left(X_{\Delta, j \Delta}\right)=\mathrm{E}\left[v_{j}\left(X_{\Delta, j \Delta}\right) \mid X_{\Delta,(j-1) \Delta}\right]+\sum_{k \geq 1} a_{j, k}\left(X_{\Delta,(j-1) \Delta}\right) \phi_{k}\left(\xi_{j}\right),
$$

\footnotetext{
${ }^{1}$ To explain this truncation we notice that $\left|a_{p, l, k}(x)\right| \leq F$ for all $x$ by the assumption.
} 
where

$$
a_{j, k}(x)=\mathrm{E}\left[v_{j}\left(X_{\Delta, j \Delta}\right) \phi_{k}\left(\xi_{j}\right) \mid X_{\Delta,(j-1) \Delta}=x\right],
$$

provided $\mathrm{E}\left[v_{j}^{2}\left(X_{\Delta, j \Delta}\right)\right]<\infty$. Representation 4.2 implies that

$$
\operatorname{Var}\left[v_{j}\left(X_{\Delta, j \Delta}\right)-M_{j} \mid X_{\Delta,(j-1) \Delta}\right]=0 \quad \text { a.s. }
$$

for

$$
M_{j}=\sum_{k \geq 1} a_{j, k}\left(X_{\Delta,(j-1) \Delta}\right) \phi_{k}\left(\xi_{j}\right)
$$

The control variates $M_{1}, \ldots, M_{J}$ cannot be used directly, since the coefficients $a_{j, k}$ are unknown and we need to truncate the summation in 4.5 to get an implementable quantity. Given that we can find implementable approximations for $M_{j}$, say $\hat{M}_{j}$, satisfying $\mathrm{E}\left[\hat{M}_{j} \mid X_{\Delta,(j-1) \Delta}\right]=0$, the idea is now to use the random variables $v_{j}\left(X_{\Delta, j \Delta}\right)-\hat{M}_{j}$ in the nested simulations step to approximate $\mathrm{E}\left[v_{j}\left(X_{\Delta, j \Delta}\right) \mid X_{\Delta,(j-1) \Delta}\right]$. Indeed, $\operatorname{Var}\left[v_{j}\left(X_{\Delta, j \Delta}\right)-\hat{M}_{j} \mid X_{\Delta,(j-1) \Delta}\right]$ will be close to zero for good approximations $\hat{M}_{j}$ (cf. (4.4)).

So first we estimate the coefficients $a_{l, k}$ by a preliminary regression using $N_{r}$ discretized paths of the process $X$ and $Q$ basis functions (see Section 3). In this way we construct the approximation of the control variate $M_{l}$ given by

$$
\hat{M}_{l, K}=\sum_{k=1}^{K} \hat{a}_{l, k}\left(X_{\Delta,(l-1) \Delta}\right) \phi_{k}\left(\xi_{l}\right)
$$

Now fix some natural numbers $N_{d}, N$ and consider the dual estimate

$$
\hat{V}_{N, N_{d}, K}=\frac{1}{N} \sum_{n=1}^{N}\left[\max _{0 \leq j \leq J}\left(g_{j}\left(X_{\Delta, j \Delta}^{(n)}\right)-\hat{Y}_{j, n, N_{d}, K}\right)\right],
$$

where

$$
\hat{Y}_{j, n, N_{d}, K}=\sum_{l=1}^{j}\left(v_{l}\left(X_{\Delta, l \Delta}^{(n)}\right)-\frac{1}{N_{d}} \sum_{n_{d}=1}^{N_{d}}\left(v_{l}\left(X_{\Delta, l \Delta}^{\left(n_{d}, n\right)}\right)-\hat{M}_{l, K}^{\left(n_{d}, n\right)}\right)\right)
$$

with

$$
\hat{M}_{l, K}^{\left(n_{d}, n\right)}=\sum_{k=1}^{K} \hat{a}_{l, k}\left(X_{\Delta,(l-1) \Delta}^{(n)}\right) \phi_{k}\left(\xi_{l}^{\left(n_{d}, n\right)}\right) .
$$

We now can prove the following result.

Theorem 4.1. Assume that all functions $v_{j}, j=1, \ldots, J$, are uniformly bounded by a constant $F$. By $\tilde{a}_{j, k}$ we denote the truncated at the level $F$ estimate defined as in (3.5), and by $\tilde{M}_{l, K}$ (resp. $\tilde{M}_{l, K}^{\left(n_{d}, n\right)}, \tilde{Y}_{j, n, N_{d}, K}, \tilde{V}_{N, N_{d}, K}$ ) the quantities defined like in (4.6) (resp. (4.9), (4.8), (4.7)) but with "hats" replaced by "tildes". Suppose that the coefficients $\left(a_{j, k}\right)$ defined in (4.3) satisfy, for all $j=1, \ldots, J$,

$$
\sum_{k=1}^{\infty} k^{\beta} \mathrm{E}\left[a_{j, k}^{2}\left(X_{\Delta,(j-1) \Delta}\right)\right] \leq B_{\beta}
$$


with some $\beta \geq 0$ and $B_{\beta}>0$ and that the basis functions $\psi_{1}, \ldots, \psi_{Q}$ are chosen in such a way that, for all $j=1, \ldots, J$ and $k \in \mathbb{N}$,

$$
\inf _{\psi \in \operatorname{span}\left(\psi_{1}, \ldots, \psi_{Q}\right)} \mathrm{E}\left[\left|a_{j, k}\left(X_{\Delta,(j-1) \Delta}\right)-\psi\left(X_{\Delta,(j-1) \Delta}\right)\right|^{2}\right] \leq D_{\kappa} Q^{-\kappa}
$$

with some $\kappa \geq 0$ and $D_{\kappa}>0$. Then it holds

$$
\begin{aligned}
\mathrm{E}\left[\left(\tilde{V}_{N, N_{d}, K}-V_{\Delta, 0}\right)^{2}\right] \leq & \frac{4 J}{N_{d}}\left(1+\frac{1}{N}\right)\left[\tilde{c} F^{2} K \frac{Q\left(\log \left(N_{r}\right)+1\right)}{N_{r}}+8 D_{\kappa} K Q^{-\kappa}+B_{\beta} K^{-\beta}\right] \\
& +\frac{4}{N} \sum_{l=1}^{J} \mathrm{E}\left[\left(v_{l}^{*}\left(X_{\Delta, l \Delta}\right)-v_{l}\left(X_{\Delta, l \Delta}\right)\right)^{2}\right]
\end{aligned}
$$

with some universal constant $\tilde{c}$.

Let us notice that the statement similar to that in Remark 3.3 applies here as well.

\subsection{Complexity analysis for fixed $J$}

Theorem 4.1 allows us to carry out complexity analysis of our algorithm. First note that the overall cost of computing the estimator $\tilde{V}_{N, N_{d}, K}$ is of order

$$
J K \max \left\{N_{r} Q^{2}, N Q, N N_{d}\right\},
$$

where the first term in 4.13 comes from the computation of the regression coefficients, the second one from the computation of $\tilde{a}_{l, k}\left(X_{\Delta,(l-1) \Delta}^{(n)}\right)$ and the last one from the computation of $\tilde{M}_{l, K}^{\left(n_{d}, n\right)}$ (other terms involved in the computation are dominated by one of these quantities). Given $\beta>0$ and $\kappa>0$ as in Theorem 4.1, we have the following constraints

$$
\max \left\{\frac{J K Q \log \left(N_{r}\right)}{N_{r} N_{d}}, \frac{J B_{\beta}}{K^{\beta} N_{d}}, \frac{J D_{\kappa} K}{Q^{\kappa} N_{d}}, \frac{J}{N}\right\} \lesssim \varepsilon^{2}
$$

to ensure the condition $\mathrm{E}\left[\left|\tilde{V}_{N, N_{d}, K}-V_{\Delta, 0}\right|^{2}\right] \lesssim \varepsilon^{2}$.

Notice that we are interested in getting the order of complexity in $\varepsilon$ as $\varepsilon \searrow 0$. To this end, we need to determine the parameters $N, N_{r}, N_{d}, K$ and $Q$ via $\varepsilon$ in such a way that the order of complexity of $\tilde{V}_{N, N_{d}, K}$ (given by (4.13)) is minimal under the constraint (4.14). Since $B_{\beta}, D_{\kappa}$ and $J$ are constants, they can be dropped from (4.13) and (4.14). Straightforward but lengthy calculations $2^{2}$ now show that the overall complexity of $\tilde{V}_{N, N_{d}, K}$ is bounded from above by

$$
C_{J, \beta, \kappa} \varepsilon^{-\frac{4(\beta+1)(\kappa+3)+4 \kappa}{(\beta+1)(\kappa+3)+\beta \kappa}} \sqrt{|\log \varepsilon|},
$$

where the constant $C_{J, \beta, \kappa}$ does not depend on $\varepsilon$. Moreover, the dependence structure in $C_{J, \beta, \kappa}$ on the parameters $\beta, \kappa$ and $J$ is given by the formula $C_{J, \beta, \kappa}=c J^{2} B_{\beta}^{3 /(1+\beta)} D_{\kappa}^{3 /(3+\kappa)}$ with some universal constant $c$. We, finally, discuss the complexity estimate 4.15).

Remark 4.2. (i) We require to choose $\beta>1$ in order to be better than the standard nested simulations approach discussed in Section 2 because $\frac{4(\beta+1)(\kappa+3)+4 \kappa}{(\beta+1)(\kappa+3)+\beta \kappa}<4$ whenever $\beta>1$.

(ii) We can achieve the complexity order $\varepsilon^{-2-\delta}$, for arbitrarily small $\delta>0$, whenever the parameters $\beta$ and $\kappa$ are sufficiently large.

(iii) In the limiting case $\kappa=0$, i.e., if the approximation error in (4.11) does not converge to 0 (e.g. due to an inappropriate choice of basis functions), we end up with the complexity of the standard nested approach of order $\varepsilon^{-4}$.

\footnotetext{
${ }^{2}$ For more detail, see Section 8
} 
In the next subsection we present the complexity analysis for the case of an increasing number of exercise dates $J \rightarrow \infty$. We also take the discretization error into account, which is the order (in $J, J \rightarrow \infty$ ) of the difference between the upper bound $V_{0}$ for the (continuous time) American option price and the upper bounds $V_{\Delta, 0}$ for the Bermudan option prices with $\Delta=T / J$.

\subsection{Complexity analysis for $J \rightarrow \infty$}

To approximate an upper bound $V_{0}$ for a true American (rather than Bermudan) option, we now let $J$ tend to infinity. We shall compare the complexities of the standard approach (the one of Section 2 applied to the discretized process) and of the regression-based approach (the one described in the beginning of Section 4).

Standard approach: Set $\Delta=T / J$, then the estimate for $V_{\Delta, 0}$ of 4.1 is

$$
V_{\Delta, N, N_{d}}=\frac{1}{N} \sum_{n=1}^{N}\left[\max _{0 \leq j \leq J}\left(g_{j}\left(X_{\Delta, j \Delta}^{(n)}\right)-Y_{\Delta, j \Delta, n, N_{d}}\right)\right]
$$

where

$$
Y_{\Delta, j \Delta, n, N_{d}}=\sum_{l=1}^{j}\left(v_{l}\left(X_{\Delta, l \Delta}^{(n)}\right)-\frac{1}{N_{d}} \sum_{n_{d}=1}^{N_{d}} v_{l}\left(X_{\Delta, l \Delta}^{\left(n_{d}, n\right)}\right)\right), \quad j=0, \ldots, J .
$$

The analogue of 2.2 takes the form

$$
\begin{aligned}
\mathrm{E}\left[\left|V_{\Delta, N, N_{d}}-V_{\Delta, 0}\right|^{2}\right] \leq & \frac{4 \sum_{l=1}^{J} \mathrm{E}\left[\operatorname{Var}\left[v_{l}\left(X_{\Delta, l \Delta}\right) \mid X_{\Delta,(l-1) \Delta}\right]\right]}{N_{d}}\left(1+\frac{1}{N}\right) \\
& +\frac{4 \sum_{l=1}^{J} \mathrm{E}\left[\left|v_{l}^{*}\left(X_{\Delta, l \Delta}\right)-v_{l}\left(X_{\Delta, l \Delta}\right)\right|^{2}\right]}{N} .
\end{aligned}
$$

Since we are considering American options in this section, the estimate $V_{\Delta, N, N_{d}}$ can be viewed as an estimate for $V_{0}$ rather than for $V_{\Delta, 0}$, i.e. this is $\mathrm{E}\left[\left|V_{\Delta, N, N_{d}}-V_{0}\right|^{2}\right]$ that should be of order $\varepsilon^{2}$ in the complexity analysis. Therefore, we need an assumption about the order of the discretization error $V_{\Delta, 0}-V_{0}$. It seems reasonably general to assume that it is of order $\frac{1}{\sqrt{J}}$. However, the discretization error might be of a different order in specific situations (see [9]). That is why we impose a more general assumption:

(A1) $V_{\Delta, 0}-V_{0}$ is of order $J^{-\alpha}$ as $J \rightarrow \infty$ with some $\alpha>0$.

We also need an assumption on the order of the second term in the right-hand side of (4.16) (which is also present in 4.12$)$ ):

$$
\sum_{l=1}^{J} \mathrm{E}\left[\left|v_{l}^{*}\left(X_{\Delta, l \Delta}\right)-v_{l}\left(X_{\Delta, l \Delta}\right)\right|^{2}\right] \text { is of order } J^{q} \text { as } J \rightarrow \infty \text { with some } q \in[0,1] \text {. }
$$

A typical-to-expect situation here is $q=1$. Another interesting variant is $q=0$ : here the strategy is to use better and better approximations $v_{l}$ for $v_{l}^{*}$ at each time point $l=1, \ldots, J$, as $J$ grows (see, e.g., Zanger [20] for bounds on $\mathrm{E}\left[\left\|v_{l}^{*}-v_{l}\right\|^{2}\right]$.) Finally, as for the first term on the right-hand side of 4.16 it is reasonable to assume only that

$$
\sum_{l=1}^{J} \mathrm{E}\left[\operatorname{Var}\left[v_{l}\left(X_{\Delta, l \Delta}\right) \mid X_{\Delta,(l-1) \Delta}\right]\right] \text { is of order } J \text { as } J \rightarrow \infty .
$$


The overall cost of computing the estimate $V_{\Delta, N, N_{d}}$ is of order $J N_{d} N$. Thus, we need to minimize this cost order under the constraint

$$
\max \left\{\frac{1}{J^{2 \alpha}}, \frac{J}{N_{d}}, \frac{J^{q}}{N}\right\} \lesssim \varepsilon^{2}
$$

which ensures that $\mathrm{E}\left[\left|V_{\Delta, N, N_{d}}-V_{0}\right|^{2}\right] \lesssim \varepsilon^{2}$ (see (4.16) and (A1)-(A3)). This leads to the complexity of $V_{\Delta, N, N_{d}}$ of order $\varepsilon^{-4-\frac{2+q}{\alpha}}$. For instance, in the case $\alpha=1 / 2, q=1$ (resp. $\alpha=1 / 2$, $q=0)$ we get the complexity $O\left(\varepsilon^{-10}\right)$ (resp. $\left.O\left(\varepsilon^{-8}\right)\right)$.

Regression-based approach: We suppose that the assumptions of Theorem 4.1 are satisfied uniformly in $J \in \mathbb{N}$ and again assume (A1) and (A2) (as for (A3), we do not need it here). The cost of computing $\tilde{V}_{N, N_{d}, K}$ is of order

$$
J K \max \left\{N_{r} Q^{2}, N Q, N N_{d}\right\} .
$$

We need to minimize this under the constraints

$$
\max \left\{\frac{1}{J^{2 \alpha}}, \frac{J K Q \log \left(N_{r}\right)}{N_{r} N_{d}}, \frac{J B_{\beta}}{K^{\beta} N_{d}}, \frac{J D_{\kappa} K}{Q^{\kappa} N_{d}}, \frac{J^{q}}{N}\right\} \lesssim \varepsilon^{2},
$$

which ensures that $\mathrm{E}\left[\left|\tilde{V}_{N, N_{d}, K}-V_{0}\right|^{2}\right] \lesssim \varepsilon^{2}$ (see 4.12) and (A1)-(A2)). Straightforward but lengthy calculation ${ }^{3}$ show that the overall complexity of $\tilde{V}_{N, N_{d}, K}$ is bounded from above by

$$
C_{\beta, \kappa} \varepsilon^{-\frac{(4 \alpha+2+q)(\beta+1)(\kappa+3)+(\beta+4 \alpha+1+q) \kappa}{\alpha(\beta+1)(\kappa+3)+\alpha \beta \kappa}} \sqrt{|\log \varepsilon|},
$$

where the constant $C_{\beta, \kappa}$ does not depend on $\varepsilon$. Moreover, the dependence on $\beta$ and $\kappa$ is described by the formula $C_{\beta, \kappa}=c B_{\beta}^{3 /(1+\beta)} D_{\kappa}^{3 /(3+\kappa)}$ with some universal constant $c$. We, finally, discuss the complexity estimate (4.17).

Remark 4.3. (i) We again require to choose $\beta>1$ in order to be better than the standard approach discussed above, because, as a straightforward calculation shows,

$$
\frac{(4 \alpha+2+q)(\beta+1)(\kappa+3)+(\beta+4 \alpha+1+q) \kappa}{\alpha(\beta+1)(\kappa+3)+\alpha \beta \kappa}<4+\frac{2+q}{\alpha}
$$

whenever $\beta>1$.

(ii) We can achieve the complexity order $\varepsilon^{-2-\frac{3+q}{2 \alpha}-\delta}$, for arbitrarily small $\delta>0$, whenever the parameters $\beta$ and $\kappa$ are sufficiently large. In particular, this gives us $O\left(\varepsilon^{-6-\delta}\right)$ (resp. $O\left(\varepsilon^{-5-\delta}\right)$ ) when $\alpha=1 / 2, q=1$ (resp. $\left.\alpha=1 / 2, q=0\right)$, which is to be compared with $O\left(\varepsilon^{-10}\right)$ (resp. $O\left(\varepsilon^{-8}\right)$ ) in the case of the standard approach.

\section{Examples and discussion of conditions}

Suppose that the process $\left(X_{t}\right)_{t \in[0, T]}$ solves the SDE

$$
d X_{t}=\mu\left(X_{t}\right) d t+\sigma\left(X_{t}\right) d W_{t}, \quad t \in[0, T],
$$

where $\mu$ and $\sigma$ are globally Lipschitz functions $\mathbb{R} \rightarrow \mathbb{R}$. Consider the Euler discretization scheme, which is of the form

$$
X_{\Delta, j \Delta}=X_{\Delta,(j-1) \Delta}+\mu\left(X_{\Delta,(j-1) \Delta}\right) \Delta+\sigma\left(X_{\Delta,(j-1) \Delta}\right) \xi_{j} \sqrt{\Delta}, \quad j=1, \ldots, J,
$$

\footnotetext{
${ }^{3}$ The calculations are similar to the derivation of 4.15 , which is discussed in Section 8 ,
} 
where $\xi_{1}, \ldots, \xi_{J}$ are independent $N(0,1)$ random variables. In this case, we have

$$
\Phi_{\Delta}(x, y)=x+\mu(x) \Delta+\sigma(x) y \sqrt{\Delta}
$$

and the orthonormal system $\left(\phi_{k}\right)_{k \in \mathbb{Z}_{+}}$in $L^{2}(\mathbb{R}, N(0,1))$ can be chosen to be the system of normalised Hermite polynomials:

$$
\phi_{k}=\frac{H_{k}}{\sqrt{k !}}, \quad H_{k}(x)=(-1)^{k} e^{x^{2} / 2} \frac{d^{k}}{d x^{k}} e^{-x^{2} / 2} .
$$

Then the coefficients $a_{j, k}$ are given by formula

$$
a_{j, k}(x)=\frac{1}{\sqrt{k !}} \mathrm{E}\left[v_{j}(x+\mu(x) \Delta+\sigma(x) \xi \sqrt{\Delta}) H_{k}(\xi)\right]
$$

with $\xi \sim N(0,1)$. To get more insight into the behaviour of $a_{j, k}$ in $k$, we need to know the structure of the approximations $v_{j}$. While we did not assume anything on their structure until now, in practice one often models $v_{j}$ as linear combinations of some basis functions, e.g. polynomials (for instance, in the Longstaff-Schwartz algorithm with a polynomial basis). Let us now verify the assumption 4.10 in a couple of particular examples.

Example 5.1. Let

$$
v_{j}(y)=\sum_{i=0}^{p} \alpha_{j, i} y^{i}, \quad j=1, \ldots, J
$$

(think of polynomial basis functions). Since, with $\xi \sim N(0,1), H_{k}(\xi)$ is orthogonal in $L^{2}$ to all polynomials in $\xi$ of degree less than $k$, it follows from (5.1) that

$$
a_{j, k} \equiv 0 \quad \text { whenever } k \geq p+1 \text {. }
$$

Then, for any $\beta>0$, there is an appropriate constant $B_{\beta}>0$ such that, for all $j=1, \ldots, J$,

$$
\sum_{k=1}^{\infty} k^{\beta} \mathrm{E}\left[a_{j, k}^{2}\left(X_{\Delta,(j-1) \Delta}\right)\right]=\sum_{k=1}^{p} k^{\beta} \mathrm{E}\left[a_{j, k}^{2}\left(X_{\Delta,(j-1) \Delta}\right)\right] \leq B_{\beta} .
$$

(Notice that, since the coefficients $\mu$ and $\sigma$ of the SDE are globally Lipschitz, all polynomial moments of the Euler discretization are finite, hence all $\mathrm{E}\left[a_{j, k}^{2}\left(X_{\Delta,(j-1) \Delta}\right)\right]$ are finite.) Thus, assumption (4.10) is satisfied and, moreover, we can take arbitrarily large $\beta>0$ (at a cost of possibly getting large $B_{\beta}$ ).

Example 5.2. Let now

$$
v_{j}(y)=\sum_{l=-p}^{p} \alpha_{j, l} \exp \{i h l y\}, \quad j=1, \ldots, J
$$

that is, at each time step $j=1, \ldots, J$ our approximations $v_{j}$ are trigonometric polynomials with period $2 \pi / h$, for some given $h>0$. With $\xi \sim N(0,1)$ we have

$$
\begin{aligned}
a_{j, k}(x) & =\frac{1}{\sqrt{k !}} \mathrm{E}\left[v_{j}(x+\mu(x) \Delta+\sigma(x) \sqrt{\Delta} \xi) H_{k}(\xi)\right] \\
& =\frac{1}{\sqrt{k !}} \sum_{l=-p}^{p} \alpha_{j, l} \exp \{i h l(x+\mu(x) \Delta)\} \mathrm{E}\left[\exp \{i h l \sigma(x) \sqrt{\Delta} \xi\} H_{k}(\xi)\right] .
\end{aligned}
$$


Using the definition of the Hermite polynomials and integrating by parts $k$ times, we compute

$$
\mathrm{E}\left[\exp \{i a \xi\} H_{k}(\xi)\right]=(i a)^{k} \exp \left\{-\frac{a^{2}}{2}\right\}
$$

Hence,

$$
\left|a_{j, k}(x)\right| \leq \frac{h^{k} \Delta^{k / 2}}{\sqrt{k !}} \sum_{l=-p}^{p}\left|\alpha_{j, l}\right| l^{k}|\sigma(x)|^{k} \exp \left\{-\frac{h^{2} l^{2} \sigma^{2}(x) \Delta}{2}\right\} .
$$

Assuming for simplicity that $\sigma$ is bounded, we get

$$
\left|a_{j, k}(x)\right| \leq \sqrt{\frac{K_{j} C^{k}}{k !}}
$$

with some positive constants $K_{j}$ and $C$. Hence, for any $\beta>0$ and for all $j=1, \ldots, J$,

$$
\sum_{k=1}^{\infty} k^{\beta} \mathrm{E}\left[a_{j, k}^{2}\left(X_{\Delta,(j-1) \Delta}\right)\right] \leq\left[\max _{j=1, \ldots, J} K_{j}\right] \sum_{k=1}^{\infty} \frac{k^{\beta} C^{k}}{k !}=: B_{\beta}<\infty .
$$

Thus, provided $\sigma$ is bounded, for arbitrarily large $\beta>0$, there exists an appropriate $B_{\beta}>0$ such that assumption (4.10) is satisfied.

\section{Numerical results}

As can be easily seen, the optimal solution for the parameter $N$ is of the same order (w.r.t. $\varepsilon$ ) both in the standard and in the regression-based approaches. Therefore, let us ignore the error term

$$
\frac{4}{N} \sum_{l=1}^{J} \mathrm{E}\left[\left|v_{l}^{*}\left(X_{\Delta, l \Delta}\right)-v_{l}\left(X_{\Delta, l \Delta}\right)\right|^{2}\right]
$$

in $(4.12)$ and (4.16). Hence, we are interested in the remaining terms

$$
\mathrm{E}\left[\operatorname{Var}\left[v_{l}\left(X_{\Delta, l \Delta}\right) \mid X_{\Delta,(l-1) \Delta}\right]\right]
$$

and

$$
\mathrm{E}\left[\operatorname{Var}\left[v_{l}\left(X_{\Delta, l \Delta}\right)-\tilde{M}_{l, K} \mid X_{\Delta,(l-1) \Delta}\right]\right],
$$

for $l=1, \ldots, J$, respectively. In terms of the numerical implementation, we will choose $N$ large enough so that (6.1) does not really affect the overall error. That is, we now consider $J$ and $N$ as fixed parameters.

Standard approach with fixed $J$ and $N$ : We recall that the overall cost of computing the estimator $V_{\Delta, N, N_{d}}$ is of order $J N_{d} N$. Since we consider only the variance terms, we set $N_{d} \asymp \varepsilon^{-2}$ to ensure that (see 4.16)

$$
\frac{4}{N_{d}}\left(1+\frac{1}{N}\right) \sum_{l=1}^{J} \mathrm{E}\left[\operatorname{Var}\left[v_{l}\left(X_{\Delta, l \Delta}\right) \mid X_{\Delta,(l-1) \Delta}\right]\right] \lesssim \varepsilon^{2} .
$$

Thus, we have for the complexity

$$
\mathcal{C}_{\text {standard }} \asymp J N_{d} N \asymp \varepsilon^{-2} .
$$


Regression-based approach with fixed $J$ and $N$ : The overall cost of computing the estimator $\tilde{V}_{N_{d}, N, K}$ is of order

$$
J K \max \left\{N_{r} Q^{2}, N N_{d}\right\} .
$$

Notice that, since $N$ is considered to be fixed, the term $N Q$ (cf. (4.13)) is dominated by $N_{r} Q^{2}$. We have the constraints

$$
\max \left\{\frac{J K Q \log \left(N_{r}\right)}{N_{r} N_{d}}, \frac{J B_{\beta}}{K^{\beta} N_{d}}, \frac{J D_{\kappa} K}{Q^{\kappa} N_{d}}\right\} \lesssim \varepsilon^{2}
$$

to ensure the condition

$$
\frac{4}{N_{d}}\left(1+\frac{1}{N}\right) \sum_{l=1}^{J} \mathrm{E}\left[\operatorname{Var}\left[v_{l}\left(X_{\Delta, l \Delta}\right)-\tilde{M}_{l, K} \mid X_{\Delta,(l-1) \Delta}\right]\right] \lesssim \varepsilon^{2} .
$$

Then, the resulting complexity bound is given by

$$
\mathcal{C}_{\text {regression }} \lesssim C_{J, N, \beta, \kappa} \varepsilon^{-\frac{2(\beta+1)(\kappa+3)+2 \kappa}{(\beta+1)(\kappa+3)+\beta \kappa}} \sqrt{|\log \varepsilon|},
$$

where $C_{J, N, \beta, \kappa}=c J^{3 / 2} N^{1 / 2} B_{\beta}^{3 /(1+\beta)} D_{\kappa}^{3 /(3+\kappa)}$ with some universal constant $c$. Notice that the complexity in $(6.9)$ is better than that in $(6.5)$ whenever $\beta>1$. Moreover, we can achieve the complexity order $\varepsilon^{-1-\delta}$ in $(6.9)$, for arbitrarily small $\delta>0$, whenever the parameters $\beta$ and $\kappa$ are sufficiently large.

In constructing the numerical experiments below, for the regression-based approach, we need to choose several values of $\varepsilon$ and the values of $N_{r}, N_{d}, K$ and $Q$ for each value of $\varepsilon$. To this end, we use the "limiting formulas" as $\beta, \kappa \rightarrow \infty$. Ignoring the remaining constants as well as the log-term for $N_{r}$, those "limiting formulas" give us $N_{r}=O\left(\varepsilon^{-1}\right), N_{d}=O\left(\varepsilon^{-1}\right), K=O(1)$ and $Q=O(1)$. In more detail, we choose the parameters for each $\varepsilon=2^{-i}, i \in\{2,3,4,5,6\}$, as follows:

$$
N=5 \cdot 10^{4}, \quad K=1, \quad Q=d+2, \quad N_{d}=8 \cdot \varepsilon^{-1}, \quad N_{r}=256 \cdot \varepsilon^{-1} .
$$

As for the basis functions, we use polynomials of $d$ variables up to degree 1 as well as the function $g_{j}$, which will be independent of $j$ (payoff of a Bermudan max-call option). Altogether $Q=d+2$ basis functions. Regarding the standard approach, we choose for each $\varepsilon=2^{-i}$, $i \in\{2,3,4,5\}$, the parameters via

$$
N=5 \cdot 10^{4}, \quad N_{d}=2 \cdot \varepsilon^{-2} .
$$

Notice that we use less values for $\varepsilon$ in case of the standard approach, since the computing time for $\varepsilon=2^{-5}$ in the standard approach is already much higher than that in the regressionbased approach for $\varepsilon=2^{-6}$, with comparable values of the estimated root mean squared errors (RMSE) $\sqrt{\mathrm{E}\left[\left|V_{\Delta, N, N_{d}}-V_{\Delta, 0}\right|^{2}\right]}$ and $\sqrt{\mathrm{E}\left[\left|\tilde{V}_{N, N_{d}, K}-V_{\Delta, 0}\right|^{2}\right]}$. In addition, we implement the multilevel approach from [6] in the following way: set $L=-\log _{2}(\varepsilon)-2$ for $\varepsilon=2^{-i}$, $i \in\{2,3,4,5\}$ and choose $\left(N_{d}\right)_{l}=48 \cdot 4^{l}$ and $N_{l}=2^{16-l}$ for $l=0, \ldots, L$. Run the multilevel algorithm until the level $L$ is reached. Thus, the cost is of order $\sum_{l=0}^{L}\left(N_{d}\right)_{l} N_{l}=O\left(2^{L}\right)=O\left(\varepsilon^{-1}\right)$, similar to the one of the regression-based approach.

Below, we compute the numerical complexities, given 500 independent simulations, and compare it with the theoretical ones, namely, $O\left(\varepsilon^{-2}\right)$ for the standard approach and $O\left(\varepsilon^{-1}\right)$ for the multilevel and regression-based approaches ("limiting formulas" as $\beta, \kappa \rightarrow \infty$ ). Note that we compute the regression estimates for $v_{j}(x)$ by means of the algorithm of Tsitsiklis and Van 
Roy (see [18], [19] or Section 8.6 in [10]), given $5 \cdot 10^{4}$ independent paths and $\frac{(d+1)(d+2)}{2}+1$ basis functions (polynomials of $d$ variables up to degree 2 as well as the function $g_{j}$ ) for all the standard, regression-based and multilevel approaches. Further, due to practical purposes, we do not allow to exercise at time $t=0$, which gives us a modified price, namely

$$
V_{\Delta, 0}=\mathrm{E}\left[\max _{1 \leq j \leq J}\left(g_{j}\left(X_{\Delta, j \Delta}\right)-Y_{\Delta, j \Delta}\right)\right]
$$

\subsection{Two-dimensional example}

We consider the following SDE for $d=m=2(Q=4)$

$$
d X_{t}^{i}=\left(r-\delta^{i}\right) X_{t}^{i} d t+\sigma^{i} X_{t}^{i} d W_{t}^{i}, \quad t \in[0,1], \quad i=1,2,
$$

where $r=0$ and $x_{0}^{i}=100, \sigma^{i}=0.2, \delta^{i}=0.02$, for $i=1,2$. Hence, $X_{t}^{1}$ and $X_{t}^{2}$ are two independent geometric Brownian motions. Further, we consider the Bermudan maxcall option with strike price 100 and 20 exercise opportunities $(J=20)$, that is, $g_{j}(x)=$ $\max \left\{\max \left\{x_{1}, x_{2}\right\}-100,0\right\}, x=\left(x_{1}, x_{2}\right)$, for all $j$. The "true" upper bound $V_{\Delta, 0} \approx 12.57$ is estimated as the mean value of 100 independent computations of $V_{\Delta, N, N_{d}}$ with $N=N_{d}=5 \cdot 10^{4}$.

As can be seen from the first plot in Figure 6.1, the estimated numerical complexity is about $\mathrm{RMSE}^{-0.84}$ for the regression-based approach, $\mathrm{RMSE}^{-1.31}$ for the standard approach and $\mathrm{RMSE}^{-0.94}$ for the multilevel approach. (We speak about numerically estimated RMSEs here.) The reason for the somewhat unexpected slope 1.31 in the standard approach is that, in this numerical example, the numerical MSE turned out to be strictly smaller than the left-hand side of (6.4), which is of course possible in specific examples. (Indeed, from the plot corresponding to the standard approach we get $\mathrm{RMSE} \asymp \varepsilon^{2 / 1.31}$, that is, MSE $\asymp \varepsilon^{4 / 1.31}$, which is smaller than const $/ N_{d} \asymp \varepsilon^{2}$.) We see that the regression-based approach works nicely, and we can save much computing time as compared to the standard and multilevel approaches to obtain similar accuracies.

\subsection{Five-dimensional example}

We consider the following SDE for $d=m=5(Q=7)$

$$
d X_{t}^{i}=\left(r-\delta^{i}\right) X_{t}^{i} d t+\sigma^{i} X_{t}^{i} A^{i} d W_{t}, \quad t \in[0,1], \quad i=1, \ldots, 5,
$$

where $r=0, x_{0}^{i}=100, \sigma^{i}=0.2, \delta^{i}=0.02 \forall i$, and $A^{i}:=\left(A^{i, 1} \cdots A^{i, 5}\right), A A^{T}=\left(\rho_{i k}\right)_{i, k=1, \ldots, 5}$ with $\rho_{i k}=\rho_{k i} \in[-1,1]$ and $\rho_{i k}=1$ for $i=k$ (that is, $A^{i} W, i=1, \ldots, 5$, are correlated Brownian motions). For $i<k$ we choose

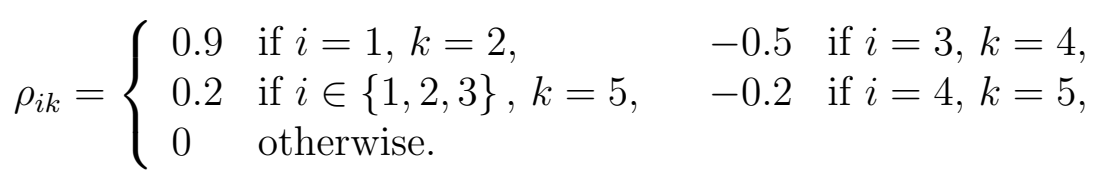

Again, we consider the Bermudan max-call option with strike price 100, but with only 10 exercise opportunities $(J=10)$, that is, $g_{j}(x)=\max \left\{\max _{i \in\{1, \ldots, 5\}} x_{i}-100,0\right\}$, for all $j$, and estimate the upper bound $V_{\Delta, 0} \approx 21.07$ via 100 independent simulations of $V_{\Delta, N, N_{d}}$ with $N=N_{d}=5 \cdot 10^{4}$.

Our empirical findings are illustrated in the second plot in Figure 6.1. We observe the numerical complexities of order $\mathrm{RMSE}^{-0.76}$ for the regression-based approach, $\mathrm{RMSE}^{-1.22}$ for the standard approach and $\mathrm{RMSE}^{-0.79}$ for the multilevel approach. Even though the numerical 

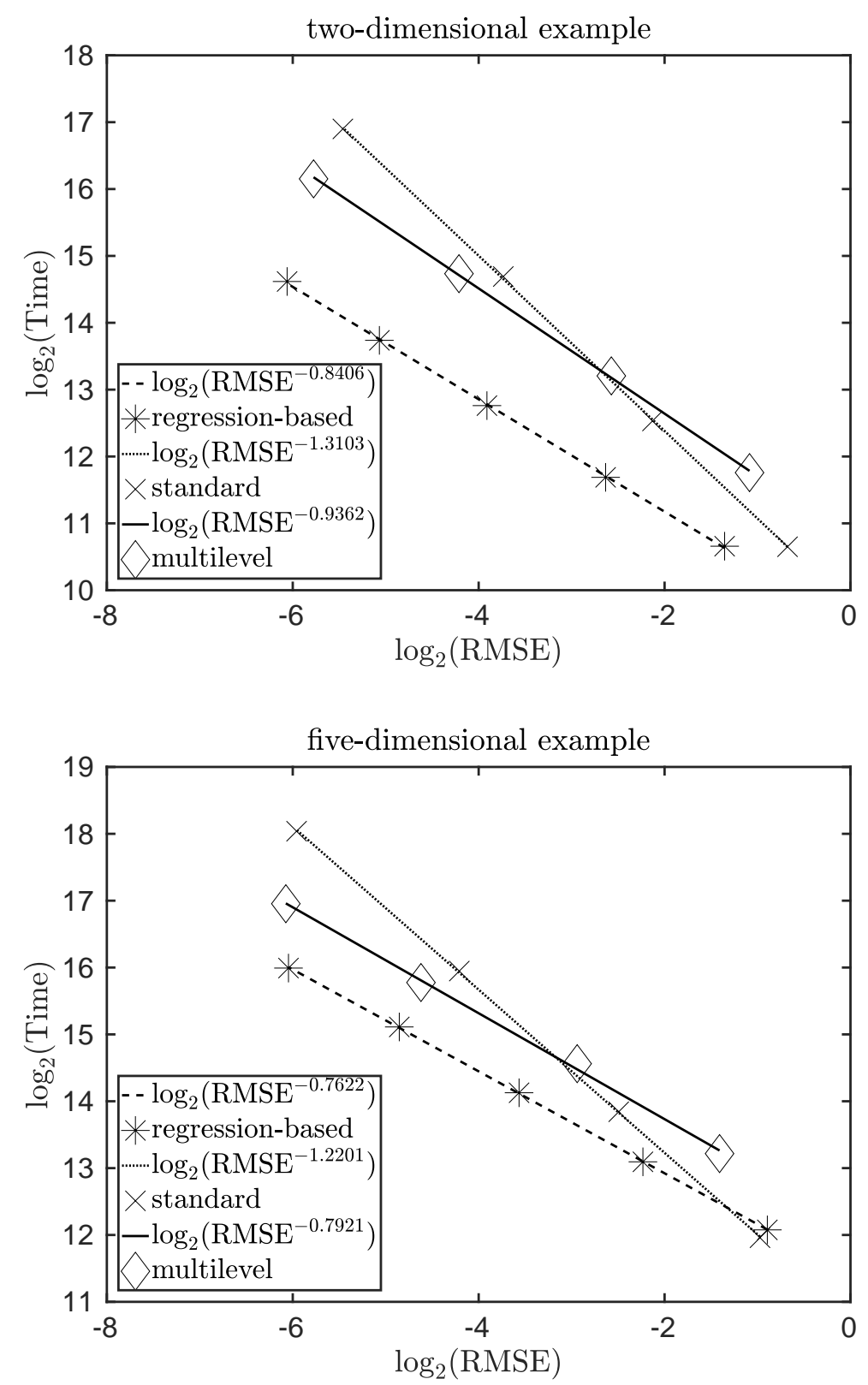

Figure 6.1: Numerical complexities of the regression-based, standard and multilevel approaches in the two- and five-dimensional cases.

complexities of the regression-based and multilevel approaches are close to each other, we observe that the computing time in case of the regression-based approach is much smaller than the multilevel one, whereas the RMSEs are in a similar region. As in the previous example, the regression-based approach shows a significant complexity reduction effect and outperforms the standard and multilevel approaches numerically. 


\section{Proofs}

\section{Proof of Theorem 2.1}

In what follows, conditioning on $X^{(n)}$ is a shorthand for conditioning on $\sigma\left(X_{j}^{(n)}, 0 \leq j \leq J\right)$.

We set

$$
Y_{j}^{(n)}:=\mathrm{E}\left[Y_{j, n, N_{d}} \mid X^{(n)}\right]
$$

and observe that

$$
Y_{j}^{(n)}=\sum_{l=1}^{j}\left(v_{l}\left(X_{l}^{(n)}\right)-\mathrm{E}\left[v_{l}\left(X_{l}^{(n)}\right) \mid X_{l-1}^{(n)}\right]\right), \quad j=0, \ldots, J
$$

in particular, the process $\left(Y_{j}^{(n)}\right)$ has the same distribution as $\left(Y_{j}\right)$. Further, we have

$$
\begin{aligned}
\mathrm{E}\left[V_{N, N_{d}} \mid X_{.}^{(n)}\right] & =\frac{1}{N} \sum_{n=1}^{N} \mathrm{E}\left[\max _{0 \leq j \leq J}\left(g_{j}\left(X_{j}^{(n)}\right)-Y_{j, n, N_{d}}\right) \mid X^{(n)}\right] \\
& \geq \frac{1}{N} \sum_{n=1}^{N} \max _{0 \leq j \leq J} \mathrm{E}\left[g_{j}\left(X_{j}^{(n)}\right)-Y_{j, n, N_{d}} \mid X^{(n)}\right] \\
& =\frac{1}{N} \sum_{n=1}^{N} \max _{0 \leq j \leq J}\left(g_{j}\left(X_{j}^{(n)}\right)-Y_{j}^{(n)}\right),
\end{aligned}
$$

which implies the required inequality $\mathrm{E} V_{N, N_{d}} \geq V_{0}$ by taking expectations of both sides.

For each $n \in\{1, \ldots, N\}$, we now introduce the filtration $\left(\overline{\mathcal{F}}_{j}^{(n)}\right)_{j \in\{0, \ldots, J\}}$ via $\overline{\mathcal{F}}_{0}^{(n)}=$ triv and $\overline{\mathcal{F}}_{j}^{(n)}=\sigma\left(X_{1}^{(n)}, \ldots, X_{j}^{(n)}, X_{1}^{\left(n_{d}, n\right)}, \ldots, X_{j}^{\left(n_{d}, n\right)}, n_{d}=1, \ldots, N_{d}\right), j \in\{1, \ldots, J\}$. Next, we have

$$
\begin{aligned}
\mathrm{E}\left[\left(V_{N, N_{d}}-V_{0}\right)^{2}\right] & =\left(\mathrm{E} V_{N, N_{d}}-V_{0}\right)^{2}+\operatorname{Var}\left[V_{N, N_{d}}\right] \\
& =\left(\mathrm{E}\left[V_{N, N_{d}}-\frac{1}{N} \sum_{n=1}^{N} \max _{0 \leq j \leq J}\left(g_{j}\left(X_{j}^{(n)}\right)-Y_{j}^{(n)}\right)\right]\right)^{2}+\operatorname{Var}\left[V_{N, N_{d}}\right] .
\end{aligned}
$$

For the first term in (7.1), that is, for the squared bias, we obtain

$$
\begin{aligned}
& \left(\mathrm{E} V_{N, N_{d}}-V_{0}\right)^{2} \\
\leq & \mathrm{E}\left[\left(V_{N, N_{d}}-\frac{1}{N} \sum_{n=1}^{N} \max _{0 \leq j \leq J}\left(g_{j}\left(X_{j}^{(n)}\right)-Y_{j}^{(n)}\right)\right)^{2}\right] \\
\leq & \frac{1}{N} \sum_{n=1}^{N} \mathrm{E}\left[\left(\max _{0 \leq j \leq J}\left(g_{j}\left(X_{j}^{(n)}\right)-Y_{j, n, N_{d}}\right)-\max _{0 \leq j \leq J}\left(g_{j}\left(X_{j}^{(n)}\right)-Y_{j}^{(n)}\right)\right)^{2}\right] \\
\leq & \frac{1}{N} \sum_{n=1}^{N} \mathrm{E} \max _{0 \leq j \leq J}\left[\left(Y_{j, n, N_{d}}-Y_{j}^{(n)}\right)^{2}\right],
\end{aligned}
$$

where we used $\left(\frac{1}{N} \sum_{n=1}^{N} a_{n}\right)^{2} \leq \frac{1}{N} \sum_{n=1}^{N} a_{n}^{2}$ in the first inequality and

$$
\left|\max _{j} a_{j}-\max _{j} b_{j}\right| \leq \max _{j}\left|a_{j}-b_{j}\right|
$$


in the second one. Since $\left(Y_{j, n, N_{d}}-Y_{j}^{(n)}\right)$ is an $\left(\overline{\mathcal{F}}_{j}^{(n)}\right)$-martingale, Doob's $L^{2}$ inequality yields

$$
\mathrm{E} \max _{0 \leq j \leq J}\left[\left(Y_{j, n, N_{d}}-Y_{j}^{(n)}\right)^{2}\right] \leq 4 \mathrm{E}\left[\left(Y_{J, n, N_{d}}-Y_{J}^{(n)}\right)^{2}\right]
$$

so that we get

$$
\left(\mathrm{E} V_{N, N_{d}}-V_{0}\right)^{2} \leq \frac{4}{N} \sum_{n=1}^{N} \mathrm{E}\left[\left(Y_{J, n, N_{d}}-Y_{J}^{(n)}\right)^{2}\right] .
$$

Proceeding as follows

$$
\begin{aligned}
\mathrm{E}\left[\left(Y_{J, n, N_{d}}-Y_{J}^{(n)}\right)^{2}\right] & =\mathrm{E}\left[\operatorname{Var}\left[Y_{J, n, N_{d}} \mid X^{(n)}\right]\right] \\
& =\mathrm{E}\left[\operatorname{Var}\left[\sum_{l=1}^{J} \frac{1}{N_{d}} \sum_{n_{d}=1}^{N_{d}} v_{l}\left(X_{l}^{\left(n_{d}, n\right)}\right) \mid X^{(n)}\right]\right] \\
& =\mathrm{E}\left[\sum_{l=1}^{J} \frac{1}{N_{d}} \operatorname{Var}\left[v_{l}\left(X_{l}^{(n)}\right) \mid X_{l-1}^{(n)}\right]\right] \\
& =\frac{1}{N_{d}} \sum_{l=1}^{J} \mathrm{E}\left[\operatorname{Var}\left[v_{l}\left(X_{l}\right) \mid X_{l-1}\right]\right],
\end{aligned}
$$

we obtain the upper bound for the squared bias

$$
\left(\mathrm{E} V_{N, N_{d}}-V_{0}\right)^{2} \leq \frac{4}{N_{d}} \sum_{l=1}^{J} \mathrm{E}\left[\operatorname{Var}\left[v_{l}\left(X_{l}\right) \mid X_{l-1}\right]\right] .
$$

Recall the almost sure property of the Doob martingale

$$
Y_{j}^{*,(1)}:=\sum_{l=1}^{j}\left(v_{l}^{*}\left(X_{l}^{(1)}\right)-\mathrm{E}\left[v_{l}^{*}\left(X_{l}^{(1)}\right) \mid X_{l-1}^{(1)}\right]\right),
$$

which here takes the form

$$
\max _{0 \leq j \leq J}\left(g_{j}\left(X_{j}^{(1)}\right)-Y_{j}^{*,(1)}\right)=v_{0}^{*}\left(X_{0}^{(1)}\right)=v_{0}^{*}\left(x_{0}\right)
$$

(see [17]), and, in particular, implies that the random variable $\max _{0 \leq j \leq J}\left(g_{j}\left(X_{j}^{(1)}\right)-Y_{j}^{*,(1)}\right)$ is, in fact, deterministic. We, therefore, derive

$$
\begin{aligned}
\operatorname{Var}\left[V_{N, N_{d}}\right] & =\frac{1}{N} \operatorname{Var}\left[\max _{0 \leq j \leq J}\left(g_{j}\left(X_{j}^{(1)}\right)-Y_{j, 1, N_{d}}\right)\right] \\
& =\frac{1}{N} \operatorname{Var}\left[\max _{0 \leq j \leq J}\left(g_{j}\left(X_{j}^{(1)}\right)-Y_{j, 1, N_{d}}\right)-\max _{0 \leq j \leq J}\left(g_{j}\left(X_{j}^{(1)}\right)-Y_{j}^{*,(1)}\right)\right] \\
& \leq \frac{1}{N} \mathrm{E} \max _{0 \leq j \leq J}\left[\left(Y_{j}^{*,(1)}-Y_{j, 1, N_{d}}\right)^{2}\right],
\end{aligned}
$$

for the second term in (7.1). Again using Doob's $L^{2}$ inequality together with the fact that 
martingale differences are uncorrelated, we get

$$
\begin{aligned}
& \operatorname{Var}\left[V_{N, N_{d}}\right] \leq \frac{4}{N} \mathrm{E}\left[\left(Y_{J}^{*,(1)}-Y_{J, 1, N_{d}}\right)^{2}\right]=\frac{4}{N} \operatorname{Var}\left[Y_{J}^{*,(1)}-Y_{J, 1, N_{d}}\right] \\
& =\frac{4}{N} \sum_{l=1}^{J} \operatorname{Var}\left[v_{l}^{*}\left(X_{l}^{(1)}\right)-v_{l}\left(X_{l}^{(1)}\right)-\mathrm{E}\left[v_{l}^{*}\left(X_{l}^{(1)}\right) \mid X_{l-1}^{(1)}\right]+\frac{1}{N_{d}} \sum_{n_{d}=1}^{N_{d}} v_{l}\left(X_{l}^{\left(n_{d}, 1\right)}\right)\right] \\
& =\frac{4}{N} \sum_{l=1}^{J} \mathrm{E}\left[\operatorname{Var}\left[v_{l}^{*}\left(X_{l}^{(1)}\right)-v_{l}\left(X_{l}^{(1)}\right)+\frac{1}{N_{d}} \sum_{n_{d}=1}^{N_{d}} v_{l}\left(X_{l}^{\left(n_{d}, 1\right)}\right) \mid X_{l-1}^{(1)}\right]\right] .
\end{aligned}
$$

Since, conditionally on $X_{l-1}^{(1)}$, the random variables $X_{l}^{(1)}, X_{l}^{(1,1)}, \ldots, X_{l}^{\left(N_{d}, 1\right)}$ are independent, we arrive at

$$
\begin{aligned}
& \operatorname{Var}\left[V_{N, N_{d}}\right] \\
& \leq \frac{4}{N} \sum_{l=1}^{J}\left(\mathrm{E}\left[\operatorname{Var}\left[v_{l}^{*}\left(X_{l}^{(1)}\right)-v_{l}\left(X_{l}^{(1)}\right) \mid X_{l-1}^{(1)}\right]\right]+\mathrm{E}\left[\operatorname{Var}\left[\frac{1}{N_{d}} \sum_{n_{d}=1}^{N_{d}} v_{l}\left(X_{l}^{\left(n_{d}, 1\right)}\right) \mid X_{l-1}^{(1)}\right]\right]\right) \\
& =\frac{4}{N} \sum_{l=1}^{J}\left(\mathrm{E}\left[\operatorname{Var}\left[v_{l}^{*}\left(X_{l}\right)-v_{l}\left(X_{l}\right) \mid X_{l-1}\right]\right]+\frac{1}{N_{d}} \mathrm{E}\left[\operatorname{Var}\left[v_{l}\left(X_{l}\right) \mid X_{l-1}\right]\right]\right) \\
& =\frac{1}{N} \frac{4}{N_{d}} \sum_{l=1}^{J} \mathrm{E}\left[\operatorname{Var}\left[v_{l}\left(X_{l}\right) \mid X_{l-1}\right]\right]+\frac{4}{N} \sum_{l=1}^{J} \mathrm{E}\left[\operatorname{Var}\left[v_{l}^{*}\left(X_{l}\right)-v_{l}\left(X_{l}\right) \mid X_{l-1}\right]\right] .
\end{aligned}
$$

Together with (7.1) and 7.2 , we obtain first inequality in $(2.2)$. The second one now follows from

$$
\mathrm{E}\left[\operatorname{Var}\left[v_{l}^{*}\left(X_{l}\right)-v_{l}\left(X_{l}\right) \mid X_{l-1}\right]\right] \leq \mathrm{E}\left[\left(v_{l}^{*}\left(X_{l}\right)-v_{l}\left(X_{l}\right)\right)^{2}\right]
$$

\section{Proof of Proposition 2.3}

We set

$$
Z_{j}^{(n)}:=\mathrm{E}\left[Z_{j, n, N_{d}} \mid X^{(n)}\right]
$$

and observe that

$$
Z_{j}^{(n)}=\mathrm{E}\left[v_{j}\left(X_{j}^{(n)}\right) \mid X_{j-1}^{(n)}\right], \quad j=1, \ldots, J
$$

We also define

$$
U_{N}:=\frac{1}{N} \sum_{n=1}^{N}\left[g_{J}\left(X_{J}^{(n)}\right)+\sum_{j=1}^{J}\left(g_{j-1}\left(X_{j-1}^{(n)}\right)-Z_{j}^{(n)}\right)^{+}\right]
$$

and notice that $\mathrm{E} U_{N}=U_{0}$. By Jensen's inequality

$$
\mathrm{E}\left[U_{N, N_{d}} \mid X^{(n)}\right] \geq U_{N}
$$

which, in turn, implies (2.5).

Next, we apply the formula

$$
\mathrm{E}\left[\left(U_{N, N_{d}}-U_{0}\right)^{2}\right]=\left(\mathrm{E} U_{N, N_{d}}-U_{0}\right)^{2}+\operatorname{Var}\left[U_{N, N_{d}}\right]
$$


and notice that the second term here is precisely the second term on the right-hand side of (2.6). It remains to prove that $\left(\mathrm{E} U_{N, N_{d}}-U_{0}\right)^{2}$ is equal to or less than the first term on the right-hand side of (2.6). To this end, we sketch the main steps as follows:

$$
\begin{aligned}
\left(\mathrm{E} U_{N, N_{d}}-U_{0}\right)^{2} & \leq \mathrm{E}\left[\left(U_{N, N_{d}}-U_{N}\right)^{2}\right] \\
& \leq \frac{1}{N} \sum_{n=1}^{N} \mathrm{E}\left[\left(\sum_{j=1}^{J}\left[\left(g_{j-1}\left(X_{j-1}^{(n)}\right)-Z_{j, n, N_{d}}\right)^{+}-\left(g_{j-1}\left(X_{j-1}^{(n)}\right)-Z_{j}^{(n)}\right)^{+}\right]\right)^{2}\right] \\
& \leq J \sum_{j=1}^{J} \mathrm{E}\left[\left(Z_{j, 1, N_{d}}-Z_{j}^{(1)}\right)^{2}\right] \\
& =J \sum_{j=1}^{J} \mathrm{E}\left[\left(\frac{1}{N_{d}} \sum_{n_{d}=1}^{N_{d}} v_{j}\left(X_{j}^{\left(n_{d}, 1\right)}\right)-Z_{j}^{(1)}\right)^{2}\right] \\
& =J \sum_{j=1}^{J} \mathrm{E}\left[\operatorname{Var}\left[\frac{1}{N_{d}} \sum_{n_{d}=1}^{N_{d}} v_{j}\left(X_{j}^{\left(n_{d}, 1\right)}\right) \mid X_{j-1}^{(1)}\right]\right] \\
& =\frac{J}{N_{d}} \sum_{j=1}^{J} \mathrm{E}\left[\operatorname{Var}\left[v_{j}\left(X_{j}\right) \mid X_{j-1}\right]\right] .
\end{aligned}
$$

This completes the proof.

\section{Proof of Theorem 3.1}

The expansion obviously holds for $p=1$ and $j=0$. Indeed, due to the orthonormality and completeness of the system $\left(\phi_{k}\right)$, we have

$$
f\left(X_{\Delta, \Delta}\right)=\mathrm{E}\left[f\left(X_{\Delta, \Delta}\right)\right]+\sum_{k \geq 1} a_{1,1, k}\left(x_{0}\right) \phi_{k}\left(\xi_{1}\right)
$$

with

$$
a_{1,1, k}\left(x_{0}\right)=\mathrm{E}\left[f\left(X_{\Delta, \Delta}\right) \phi_{k}\left(\xi_{1}\right)\right],
$$

provided $\mathrm{E}\left[\left|f\left(X_{\Delta, \Delta}\right)\right|^{2}\right]<\infty$. Recall that $\mathcal{G}_{l}=\sigma\left(\xi_{1}, \ldots, \xi_{l}\right), l=1,2, \ldots, L$, and $\mathcal{G}_{0}=$ triv. Suppose that 3.2 holds for $p=q$, all $j<q$, and all Borel-measurable functions $f$ with $\mathrm{E}\left[\left|f\left(X_{\Delta, q \Delta}\right)\right|^{2}\right]<\infty$. Let us prove it for $p=q+1$. Given $f$ with $\mathrm{E}\left[\left|f\left(X_{\Delta, p \Delta}\right)\right|^{2}\right]<\infty$, due to the orthonormality and completeness of the system $\left(\phi_{k}\right)$, we get by conditioning on $\mathcal{G}_{q}$,

$$
f\left(X_{\Delta, p \Delta}\right)=\mathrm{E}\left[f\left(X_{\Delta, p \Delta}\right) \mid \mathcal{G}_{q}\right]+\sum_{k \geq 1} \alpha_{p, q+1, k} \phi_{k}\left(\xi_{q+1}\right),
$$

where

$$
\alpha_{p, q+1, k}=\mathrm{E}\left[f\left(X_{\Delta, p \Delta}\right) \phi_{k}\left(\xi_{q+1}\right) \mid \mathcal{G}_{q}\right] .
$$

By the Markov property of $\left(X_{\Delta, l \Delta}\right)$, we have $\mathrm{E}\left[f\left(X_{\Delta, p \Delta}\right) \mid \mathcal{G}_{q}\right]=\mathrm{E}\left[f\left(X_{\Delta, p \Delta}\right) \mid X_{\Delta, q \Delta}\right]$. Furthermore, a calculation involving intermediate conditioning on $\mathcal{G}_{q+1}$ and the recurrence relation $X_{\Delta,(q+1) \Delta}=\Phi_{q+1}\left(X_{\Delta, q \Delta}, \xi_{q+1}\right)$ verifies that

$$
\alpha_{p, q+1, k}=\mathrm{E}\left[f\left(X_{\Delta, p \Delta}\right) \phi_{k}\left(\xi_{q+1}\right) \mid X_{\Delta, q \Delta}\right]=a_{p, q+1, k}\left(X_{\Delta, q \Delta}\right)
$$

for suitably chosen Borel-measurable functions $a_{p, q+1, k}$. We thus arrive at

$$
f\left(X_{\Delta, p \Delta}\right)=\mathrm{E}\left[f\left(X_{\Delta, p \Delta}\right) \mid X_{\Delta, q \Delta}\right]+\sum_{k \geq 1} a_{p, q+1, k}\left(X_{\Delta, q \Delta}\right) \phi_{k}\left(\xi_{q+1}\right),
$$


which is the required statement in the case $j=q$. Now assume $j<q$. The random variable $\mathrm{E}\left[f\left(X_{\Delta, p \Delta}\right) \mid X_{\Delta, q \Delta}\right]$ is square integrable and has the form $g\left(X_{\Delta, q \Delta}\right)$, hence the induction hypothesis applies, and we get

$$
\mathrm{E}\left[f\left(X_{\Delta, p \Delta}\right) \mid X_{\Delta, q \Delta}\right]=\mathrm{E}\left[f\left(X_{\Delta, p \Delta}\right) \mid X_{\Delta, j \Delta}\right]+\sum_{k \geq 1} \sum_{l=j+1}^{q} a_{p, l, k}\left(X_{\Delta,(l-1) \Delta}\right) \phi_{k}\left(\xi_{l}\right)
$$

with

$$
\begin{aligned}
a_{p, l, k}\left(X_{\Delta,(l-1) \Delta}\right) & =\mathrm{E}\left[\mathrm{E}\left[f\left(X_{\Delta, p \Delta}\right) \mid \mathcal{G}_{q}\right] \phi_{k}\left(\xi_{l}\right) \mid \mathcal{G}_{l-1}\right]=\mathrm{E}\left[f\left(X_{\Delta, p \Delta}\right) \phi_{k}\left(\xi_{l}\right) \mid \mathcal{G}_{l-1}\right] \\
& =\mathrm{E}\left[f\left(X_{\Delta, p \Delta}\right) \phi_{k}\left(\xi_{l}\right) \mid X_{\Delta,(l-1) \Delta}\right] .
\end{aligned}
$$

Formulas 7.4 and 7.5 conclude the proof.

\section{Proof of Theorem 3.2}

It holds

$$
\mathrm{E}\left[\operatorname{Var}\left[f\left(X_{\Delta, p \Delta}\right)-\tilde{M}_{j, p, K} \mid X_{\Delta, j \Delta}\right]\right]=\mathrm{E}\left[\left|M_{j, p}-\tilde{M}_{j, p, K}\right|^{2}\right]
$$

We have

$$
\begin{aligned}
\mathrm{E}\left[\left|M_{j, p}-\tilde{M}_{j, p, K}\right|^{2}\right]= & \mathrm{E}\left[\left|\sum_{k=K+1}^{\infty} \sum_{l=j+1}^{p} a_{p, l, k}\left(X_{\Delta,(l-1) \Delta}\right) \phi_{k}\left(\xi_{l}\right)\right|^{2}\right] \\
& +\mathrm{E}\left[\left|\sum_{k=1}^{K} \sum_{l=j+1}^{p}\left(a_{p, l, k}\left(X_{\Delta,(l-1) \Delta}\right)-\tilde{a}_{p, l, k}\left(X_{\Delta,(l-1) \Delta}\right)\right) \phi_{k}\left(\xi_{l}\right)\right|^{2}\right] \\
= & \sum_{k=K+1}^{\infty} \sum_{l=j+1}^{p} \mathrm{E}\left[a_{p, l, k}^{2}\left(X_{\Delta,(l-1) \Delta}\right)\right] \\
& +\sum_{k=1}^{K} \sum_{l=j+1}^{p} \mathrm{E}\left[\left(a_{p, l, k}\left(X_{\Delta,(l-1) \Delta}\right)-\tilde{a}_{p, l, k}\left(X_{\Delta,(l-1) \Delta}\right)\right)^{2}\right]
\end{aligned}
$$

It follows from Theorem 11.3 in [11] that

$$
\begin{aligned}
& \mathrm{E}\left[\left(a_{p, l, k}\left(X_{\Delta,(l-1) \Delta}\right)-\tilde{a}_{p, l, k}\left(X_{\Delta,(l-1) \Delta}\right)\right)^{2}\right] \\
& \leq \tilde{c} F^{2} \frac{Q\left(\log \left(N_{r}\right)+1\right)}{N_{r}}+8 \inf _{\psi \in \operatorname{span}\left(\psi_{1}, \ldots, \psi_{Q}\right)} \mathrm{E}\left[\left|a_{p, l, k}\left(X_{\Delta,(l-1) \Delta}\right)-\psi\left(X_{\Delta,(l-1) \Delta}\right)\right|\right]^{2}
\end{aligned}
$$

for some universal constant $\tilde{c}$, since

$$
\operatorname{Var}\left[f\left(X_{\Delta, p \Delta}\right) \phi_{k}\left(\xi_{l}\right) \mid X_{\Delta,(l-1) \Delta}=x\right] \leq F^{2}
$$

and

$$
\left|\mathrm{E}\left[f\left(X_{\Delta, p \Delta}\right) \phi_{k}\left(\xi_{l}\right) \mid X_{\Delta,(l-1) \Delta}=x\right]\right| \leq F .
$$

The result now follows from $(3.6)-(3.7)$ and $(7.6)-(7.8)$. 


\section{Proof of Theorem 4.1}

By the same calculation as the one leading to 2.2) (see the proof of Theorem 2.1), we get

$$
\begin{aligned}
\mathrm{E}\left[\left(\tilde{V}_{N, N_{d}, K}-V_{\Delta, 0}\right)^{2}\right] \leq & \frac{4}{N_{d}}\left(1+\frac{1}{N}\right) \sum_{l=1}^{J} \mathrm{E}\left[\operatorname{Var}\left[v_{l}\left(X_{\Delta, l \Delta}\right)-\tilde{M}_{l, K} \mid X_{\Delta,(l-1) \Delta}\right]\right] \\
& +\frac{4}{N} \sum_{l=1}^{J} \mathrm{E}\left[\left(v_{l}^{*}\left(X_{\Delta, l \Delta}\right)-v_{l}\left(X_{\Delta, l \Delta}\right)\right)^{2}\right] .
\end{aligned}
$$

It remains to apply Theorem 3.2 to the first term on the right-hand side.

\section{Appendix: derivation of complexity 4.15)}

Let us, for simplicity, first ignore the $\log \left(N_{r}\right)$-term in 4.14$)$ and consider only the terms w.r.t. the variables $K, Q, N, N_{d}, N_{r}$ which shall be optimized, since the constants $J, B_{\beta}, D_{\kappa}$ do not affect the terms on $\varepsilon$. Further, we consider the log-cost and log-constraints rather than (4.13) and (4.14). Let us subdivide the optimization problem into three cases:

a) $\max \left\{N_{r} Q^{2}, N Q, N N_{d}\right\}=N_{r} Q^{2}$. Here, we have the Lagrange function

$$
\begin{aligned}
L\left(K, Q, N, N_{d}, N_{r}\right):= & \log (K)+\log \left(N_{r}\right)+2 \log (Q)+\lambda_{1}\left(\log (N)-\log \left(N_{r}\right)-\log (Q)\right) \\
& +\lambda_{2}\left(\log (N)+\log \left(N_{d}\right)-\log \left(N_{r}\right)-2 \log (Q)\right) \\
& +\lambda_{3}\left(\log (K)+\log (Q)-\log \left(N_{d}\right)-\log \left(N_{r}\right)-2 \log (\varepsilon)\right) \\
& +\lambda_{4}\left(-\log \left(N_{d}\right)-\beta \log (K)-2 \log (\varepsilon)\right) \\
& +\lambda_{5}\left(\log (K)-\log \left(N_{d}\right)-\kappa \log (Q)-2 \log (\varepsilon)\right) \\
& +\lambda_{6}(-\log (N)-2 \log (\varepsilon)) .
\end{aligned}
$$

Considering $\frac{\partial L}{\partial K}=\frac{\partial L}{\partial Q}=\frac{\partial L}{\partial N}=\frac{\partial L}{\partial N_{d}}=\frac{\partial L}{\partial N_{r}}=0$ leads to the unique solution $\lambda_{1}=0, \lambda_{i}>0$ for $i>1$. More precisely, due to five equations with six variables $\lambda_{1}, \ldots, \lambda_{6}$, at least one $\lambda_{i}$ has to be zero. In the others cases $\lambda_{i}=0$ for $i>1$, we either obtain $\lambda_{k}<0$ for some $k \neq i$, or the corresponding constraint to $\lambda_{i}$ is not satisfied (that is $>0$ ) such that these solutions are not optimal, respectively they do not satisfy all constraints. Since we have $\lambda_{i}>0$ for all $i>1$, all constraints corresponding to those $\lambda_{i}$ have to be active (that is, zero). This gives us

$$
\begin{array}{r}
K=O\left(\varepsilon^{-\frac{4 \kappa}{3 \beta+\kappa+2 \beta \kappa+3}}\right), \quad Q=O\left(\varepsilon^{-\frac{4(1+\beta)}{3 \beta+\kappa+2 \beta \kappa+3}}\right), \quad N=O\left(\varepsilon^{-2}\right), \\
N_{d}=O\left(\varepsilon^{-\frac{6(1+\beta)+2 \kappa}{3 \beta+\kappa+2 \beta \kappa+3}}\right), \quad N_{r}=O\left(\varepsilon^{-\frac{4(1+\beta)(1+\kappa)}{3 \beta+\kappa+2 \beta \kappa+3}}\right),
\end{array}
$$

provided that $\beta>1$. Hence, the complexity is

$$
\mathcal{C}=O\left(K N_{r} Q^{2}\right)=O\left(\varepsilon^{-\frac{4(1+\beta)(3+\kappa)+4 \kappa}{3 \beta+\kappa+2 \beta \kappa+3}}\right) .
$$

b) $\max \left\{N_{r} Q^{2}, N Q, N N_{d}\right\}=N Q$. Here, we obtain, similarly to case a), the complexity of order

$$
O\left(\varepsilon^{-2-\frac{4(\beta+\kappa+1)}{\beta(\kappa+1)+1}}\right)
$$

for $\beta>1$, which is worse than 8.2 .

\footnotetext{
${ }^{4}$ The condition $\beta>1$ is required to satisfy $\lambda_{i}>0$ for all $i>1$.
} 
c) $\max \left\{N_{r} Q^{2}, N Q, N N_{d}\right\}=N N_{d}$. This gives us the same result as in case a). Hence, the complexity in $(8.2)$ is the overall optimal solution.

In the case $\beta=1$, we get (compare with 4.14 )

$$
N \gtrsim \varepsilon^{-2}, \quad K N_{d} \gtrsim \varepsilon^{-2},
$$

and thus

$$
\mathcal{C} \gtrsim K N N_{d} \gtrsim \varepsilon^{-4}
$$

which means that the complexity is always worse than the one of the standard nested simulations approach discussed in Section 2. Next we consider also the remaining terms $J, B_{\beta}, D_{\kappa}$ and arrive at (4.15) via equalizing all constraints in (4.14) as well as considering $N N_{d}=N_{r} Q^{2}$ (provided that $\beta>1$ ). Finally, we add the log-term concerning $\varepsilon$ in the parameters $N_{r}$ and $N_{d}$ to ensure that all constraints are really satisfied. Notice that the constant $C_{J, \beta, \kappa}$ in 4.15 is an upper bound of the constant which arises from equalizing the above mentioned constraints.

\section{References}

[1] L. Andersen and M. Broadie. Primal-dual simulation algorithm for pricing multidimensional American options. Management Science, 50(9):1222-1234, 2004.

[2] D. Belomestny, C. Bender, and J. Schoenmakers. True upper bounds for Bermudan products via non-nested Monte Carlo. Mathematical Finance, 19(1):53-71, 2009.

[3] D. Belomestny, S. Häfner, T. Nagapetyan, and M. Urusov. Variance reduction for discretised diffusions via regression. Preprint, arXiv:1510.03141v4, 2017.

[4] D. Belomestny, G. Milstein, and V. Spokoiny. Regression methods in pricing American and Bermudan options using consumption processes. Quant. Finance, 9(3):315-327, 2009.

[5] D. Belomestny and G. N. Milstein. Monte Carlo evaluation of American options using consumption processes. International Journal of Theoretical and Applied Finance, 9(04):455-481, 2006.

[6] D. Belomestny, J. Schoenmakers, and F. Dickmann. Multilevel dual approach for pricing American style derivatives. Finance and Stochastics, 17(4):717-742, 2013.

[7] N. Chen and P. Glasserman. Additive and multiplicative duals for American option pricing. Finance and Stochastics, 11(2):153-179, 2007.

[8] E. Clément, D. Lamberton, and P. Protter. An analysis of a least squares regression method for American option pricing. Finance Stoch., 6(4):449-471, 2002.

[9] P. Dupuis and H. Wang. On the convergence from discrete to continuous time in an optimal stopping problem. The Annals of Applied Probability, 15(2):1339-1366, 2005.

[10] P. Glasserman. Monte Carlo methods in financial engineering, volume 53. Springer Science \& Business Media, 2003.

[11] L. Györfi, M. Kohler, A. Krzyżak, and H. Walk. A distribution-free theory of nonparametric regression. Springer Series in Statistics. Springer-Verlag, New York, 2002. 
[12] M. B. Haugh and L. Kogan. Pricing American options: a duality approach. Oper. Res., $52(2): 258-270,2004$.

[13] P. E. Kloeden and E. Platen. Numerical solution of stochastic differential equations, volume 23. Springer Science \& Business Media, 1992.

[14] F. A. Longstaff and E. S. Schwartz. Valuing American options by simulation: A simple least-squares approach. Review of Financial studies, 14(1):113-147, 2001.

[15] G. N. Milstein and M. V. Tretyakov. Stochastic numerics for mathematical physics. Scientific Computation. Springer-Verlag, Berlin, 2004.

[16] L. C. G. Rogers. Monte Carlo valuation of American options. Math. Finance, 12(3):271$286,2002$.

[17] J. Schoenmakers, J. Zhang, and J. Huang. Optimal dual martingales, their analysis, and application to new algorithms for Bermudan products. SIAM J. Financial Math., 4(1):86116, 2013.

[18] J. N. Tsitsiklis and B. Van Roy. Optimal stopping of Markov processes: Hilbert space theory, approximation algorithms, and an application to pricing high-dimensional financial derivatives. IEEE Transactions on Automatic Control, 44(10):1840-1851, 1999.

[19] J. N. Tsitsiklis and B. Van Roy. Regression methods for pricing complex American-style options. IEEE Transactions on Neural Networks, 12(4):694-703, 2001.

[20] D. Z. Zanger. Quantitative error estimates for a least-squares Monte Carlo algorithm for American option pricing. Finance and Stochastics, 17(3):503-534, 2013. 